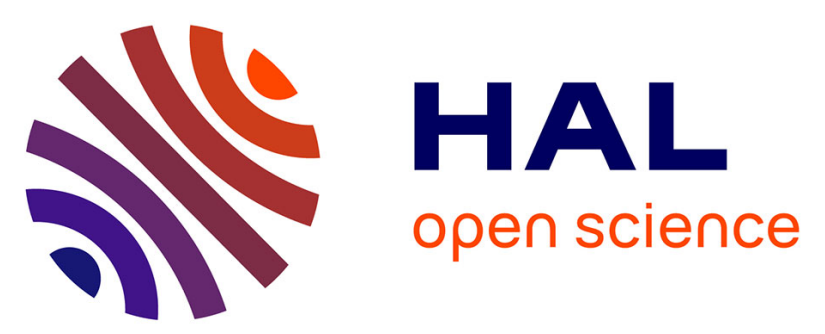

\title{
Evolutionary conservation and functional implications of circular code motifs in eukaryotic genomes
}

\author{
Gopal Dila, Christian C. Michel, Olivier Poch, Raymond Ripp, Julie D
}

Thompson

\section{- To cite this version: \\ Gopal Dila, Christian C. Michel, Olivier Poch, Raymond Ripp, Julie D Thompson. Evolutionary conservation and functional implications of circular code motifs in eukaryotic genomes. BioSystems, 2019, 175, pp.57-74. 10.1016/j.biosystems.2018.10.014 . hal-02381474}

\section{HAL Id: hal-02381474 \\ https://hal.science/hal-02381474}

Submitted on 4 Dec 2020

HAL is a multi-disciplinary open access archive for the deposit and dissemination of scientific research documents, whether they are published or not. The documents may come from teaching and research institutions in France or abroad, or from public or private research centers.
L'archive ouverte pluridisciplinaire HAL, est destinée au dépôt et à la diffusion de documents scientifiques de niveau recherche, publiés ou non, émanant des établissements d'enseignement et de recherche français ou étrangers, des laboratoires publics ou privés. 


\section{Evolutionary conservation and functional implications of circular code motifs in eukaryotic genomes}

Gopal Dila, Christian J. Michel, Olivier Poch, Raymond Ripp, Julie D. Thompson

CSTB, ICube

CNRS, University of Strasbourg

300 Boulevard Sébastien Brant

67400 Illkirch, France

Email: d.gopal@outlook.com, c.michel@unistra.fr, olivier.poch@unistra.fr, raymond.ripp@unistra.fr, thompson@unistra.fr

Keywords: circular code motifs; genome evolution; genetic code; gene expression. 


\begin{abstract}
A set $X$ of 20 trinucleotides has been found to have the highest average occurrence in the reading frame, compared to the two shifted frames, of genes of bacteria, archaea, eukaryotes, plasmids and viruses (Michel, 2017, 2015; Arquès and Michel, 1996). This set $X$ has an interesting mathematical property, since $X$ is a maximal $C^{3}$ self-complementary trinucleotide circular code (Arquès and Michel, 1996). Furthermore, any motif obtained from this circular code $X$ has the capacity to retrieve, maintain and synchronize the reading frame in genes. In a recent study of the $X$ motifs in the complete genome of the yeast, Saccharomyces cerevisiae, it was shown that they are significantly enriched in the reading frame of the genes (protein-coding regions) of the genome (Michel et al., 2017). It was suggested that these $X$ motifs may be evolutionary relics of a primitive code originally used for gene translation. The aim of this paper is to address two questions: are $X$ motifs conserved during evolution? and do they continue to play a functional role in the processes of genome decoding and protein production? In a large scale analysis involving complete genomes from four mammals and nine different yeast species, we highlight specific evolutionary pressures on the $X$ motifs in the genes of all the genomes, and identify important new properties of $X$ motif conservation at the level of the encoded amino acids. We then compare the occurrence of $X$ motifs with existing experimental data concerning protein expression and protein production, and report a significant correlation between the number of $X$ motifs in a gene and increased protein abundance. In a general way, this work suggests that motifs from circular codes, i.e. motifs having the property of reading frame retrieval, may represent functional elements located within the coding regions of extant genomes.
\end{abstract}

\title{
1. Introduction
}

The same set $X$ of trinucleotides (also known as codons) was identified in average in genes (reading frame) of bacteria, archaea, eukaryotes, plasmids and viruses (Michel 2017, 2015; Arquès and Michel, 1996). It contains the 20 following trinucleotides

$$
\begin{array}{r}
X=\{A A C, A A T, A C C, A T C, A T T, C A G, C T C, C T G, G A A, G A C, \\
\quad G A G, G A T, G C C, G G C, G G T, G T A, G T C, G T T, T A C, T T C\}
\end{array}
$$

and codes the 12 following amino acids (three and one letter notation)

$$
\begin{aligned}
X= & \{\text { Ala }, \text { Asn }, \text { Asp }, \text { Gln }, \text { Glu, Gly, Ile, Leu, Phe,Thr, Tyr }, \text { Val }\} \\
& =\{A, N, D, Q, E, G, I, L, F, T, Y, V\} .
\end{aligned}
$$

This set $X$ has several strong mathematical properties. In particular, it is self-complementary, i.e. 10 trinucleotides of $X$ are complementary to the other 10 trinucleotides of $X$, e.g. $A A C \in X$ is complementary to GTT $\in X$, and it is a circular code. A circular code is defined as a set of words such that any motif obtained from this set, allows to retrieve, maintain and synchronize the original (construction) frame. Thus, the circular code $X$ may represent a self-correcting property of the genetic code. Indeed, it has been proposed recently that the circular code $X$ may participate in the regulation of gene transcription (El Houmami and Seligmann, 2017). Other correction properties of the genetic code 
have also been proposed with roles in gene translation, including compensation of tRNA misloading (Seligmann, 2011), prevention of protein misfolding (Seligmann and Warthi, 2017), or termination of translation after ribosomal frameshifting (Seligmann and Pollock, 2004).

Motifs from the circular code $X$ (denoted (1) above) having this frame retrieval property are called $X$ motifs. Since 1996, the theory of circular codes in genes has mainly been developed by analysing the classes, the numbers and the mathematical properties of circular codes using probability-statistics, combinatorics and graph theory (reviews in Michel, 2008, and Fimmel and Strüngmann, 2018). More recently, the circular code theory was applied to the complete genome sequence of a living organism, namely the eukaryote Saccharomyces cerevisiae (Michel et al., 2017). It was shown that $X$ motifs from the circular code $X$ (1) were significantly enriched in the genes (protein-coding regions) of the genome. The authors hypothesized that the $X$ motifs may be evolutionary relics of a primitive code originally used for translation.

In this article, we describe a large-scale study of the $X$ motifs in two independent sets of complete genomes. The first set is composed of four mammal genomes, representing highly evolved species and closely related genomes. The second set is built from nine yeast genomes, representing the simplest eukaryotes with more divergent genome sequences. Each set includes a well-studied and annotated 'reference' genome: the human genome for the first set and the Saccharomyces cerevisiae genome for the second set. We first highlight specific evolutionary pressures on the $X$ motifs in the genes of both sets of genomes, and identify important new properties of $X$ motif conservation at the level of the encoded amino acids. Thus, the 20 trinucleotides of the circular code $X$ (1) are grouped according to the amino acids they encode, leading to a new hypothesis for the evolution of the genetic code where each amino acid was coded by the most constrained circular codes, namely strong comma-free and comma-free codes.

Then, we investigate the potential functional role of $X$ motifs in the regulation of gene expression. To achieve this, we compare the occurrence of $X$ motifs with existing experimental data concerning protein expression and production, and report a significant correlation between the number of $X$ motifs in a gene and increased protein abundance. Taken together, the results represent compelling evidence suggesting that $X$ motifs may indeed contribute to the complex mechanisms of protein synthesis in extant genomes.

\section{Method}

After recalling a few basic definitions of circular codes, we define three classes of motifs: the $X$ motifs constructed from the circular code $X$ (1) identified in genes, as well as non- $X$ motifs and random motifs used to evaluate the significance of $X$ motifs. The statistical analyses of these motifs are based on very simple statistics, namely frequencies and mean frequencies, leading to clear biological results. 


\subsection{Definitions of circular code}

We recall a few definitions without detailed explanation (i.e. without examples and figures) that are necessary for understanding the main properties of the $X$ motifs obtained from the trinucleotide circular code $X$ identified in genes (Michel, 2017, 2015; Arquès and Michel, 1996).

Notation 1. Let us denote the nucleotide 4-letter alphabet $B=\{A, C, G, T\}$ where $A$ stands for adenine, $C$ stands for cytosine, $G$ stands for guanine and $T$ stands for thymine. The trinucleotide set over $B$ is denoted by $B^{3}=\{A A A, \ldots, T T T\}$. The set of non-empty words (words, respectively) over $B$ is denoted by $B^{+}\left(B^{*}\right.$, respectively).

Definition 1. A set $S \subseteq B^{+}$is a code if, for each $x_{1}, \ldots, x_{n}, y_{1}, \ldots, y_{m} \in S, n, m \geq 1$, the condition $x_{1} \cdots x_{n}=$ $y_{1} \cdots y_{m}$ implies $n=m$ and $x_{i}=y_{i}$ for $i=1, \ldots, n$.

Definition 2. Any non-empty subset of the code $B^{3}$ is a code and called trinucleotide code.

Definition 3. The genetic code is a trinucleotide code. It defines a surjective map $g: \tilde{B}^{3} \rightarrow P$ where $\tilde{B}^{3}=$ $B^{3} \backslash\{T A A, T A G, T G A\}$ and $P$ is the set of the 20 peptide components (amino acids). We also use the following notation: a sequence $s$ of trinucleotides, i.e. a gene, codes a sequence noted $g(s)$ of amino acids, i.e. a protein.

Example 1. $g(G G A)=G l y, g^{-1}(G l y)=\{G G A, G G C, G G G, G G T\}$ and $g(G A C A T C C T G)=D I L$ where $D, I$ and $L$ are amino acids.

Definition 4. A trinucleotide code $X \subseteq B^{3}$ is circular if, for each $x_{1}, \ldots, x_{n}, y_{1}, \ldots, y_{m} \in X, n, m \geq 1, r \in$ $B^{*}, s \in B^{+}$, the conditions $s x_{2} \cdots x_{n} r=y_{1} \cdots y_{m}$ and $x_{1}=r s$ imply $n=m, r=\varepsilon$ (empty word) and $x_{i}=$ $y_{i}$ for $i=1, \ldots, n$.

We briefly recall the proof used to determine whether a code is circular or not, with the most recent and powerful approach which relates an oriented (directed) graph to a trinucleotide code.

Definition 5. (Fimmel et al., 2016). Let $X \subseteq B^{3}$ be a trinucleotide code. The directed graph $\mathcal{G}(X)=$ $(V(X), E(X))$ associated with $X$ has a finite set of vertices $V(X)$ and a finite set of oriented edges $E(X)$ (ordered pairs $[v, w]$ where $v, w \in X$ ) defined as follows:

$$
\left\{\begin{array}{c}
V(X)=\left\{N_{1}, N_{3}, N_{1} N_{2}, N_{2} N_{3}: N_{1} N_{2} N_{3} \in X\right\} \\
E(X)=\left\{\left[N_{1}, N_{2} N_{3}\right],\left[N_{1} N_{2}, N_{3}\right]: N_{1} N_{2} N_{3} \in X\right\}
\end{array}\right.
$$

The theorem below gives a relation between a trinucleotide code which is circular and its associated graph.

Theorem 1. (Fimmel et al., 2016). Let $X \subseteq B^{3}$ be a trinucleotide code. The following statements are equivalent:

(i) The code $X$ is circular.

(ii) The graph $\mathcal{G}(X)$ is acyclic.

We also recall the results that characterize the comma-free codes and the strong comma-free codes by the longest paths in their associated graphs.

Theorem 2. (Fimmel et al., 2016). Let $X \subseteq B^{3}$ be a trinucleotide circular code. The following statements are equivalent:

(i) $X$ is comma-free. 
(ii) The longest path in $\mathcal{G}(X)$ is of length at most 2.

Theorem 3. (Fimmel et al., 2017). Let $X \subseteq B^{3}$ be a trinucleotide circular code. The following statements are equivalent:

(i) $X$ is strong comma-free.

(ii) The longest path in $\mathcal{G}(X)$ is of length at most 1.

Thus, the reading frame is retrieved after the reading of 2 nucleotides with motifs from a strong commafree code, of 3 nucleotides ( 1 trinucleotide) with motifs from a comma-free code and of at most 13 nucleotides ( 4 trinucleotides +1 nucleotide) with motifs from circular codes.

The trinucleotide set $X$ coding the reading frame in genes of bacteria, archaea, eukaryotes, plasmids and viruses is a maximal (20 trinucleotides) $C^{3}$ (the 2 shifted codes by permutation of $X$ are also circular) self-complementary (10 trinucleotides of $X$ are complementary to the 10 other trinucleotides of $X$ ) trinucleotide circular code (Michel 2017, 2015; Arquès and Michel, 1996).

\subsection{Definition of $X$ motifs, non- $X$ motifs and random motifs}

Definition 6. As in Michel et al. (2017), a $X$ motif $m(X)$ constructed from the circular code $X$ (1), is a word with cardinality $4 \leq c \leq 20$ trinucleotides and length $l \geq c \geq 4$ trinucleotides. Here, we consider only the $X$ motifs $m(X)$ found in reading frame of genes.

Indeed, the $X$ motifs $m(X)$ have a cardinality $c \leq 20$ trinucleotides as the circular code $X$ (1) has 20 trinucleotides. The minimal length $l=4$ trinucleotides was chosen based on the requirement for 13 nucleotides in order to retrieve the reading frame. The class of motifs of $X$ with cardinality $c<4$ are excluded here because they are mostly associated with the "pure" trinucleotide repeats often found in non-coding regions of the genome (Michel et al., 2017; El Soufi and Michel, 2017).

The fundamental property of a $X$ motif $m(X)$ is the ability to retrieve, synchronize and maintain the reading frame. Indeed, a window of 13 nucleotides located anywhere in a sequence generated from the circular code $X$ (1) is sufficient to retrieve the reading (correct, construction) frame of the sequence. It is important to stress again that this window for retrieving the reading frame in a sequence can be located anywhere in the sequence, i.e. no other frame signal, including start and stop trinucleotides, is required to identify the reading frame.

Example 2. For the convenience of the reader, we give an example of a $X$ motif $m(X)=m_{1}$ from the circular code $X(1)$ in a sequence $s=\cdots A A A G G T G C C G A A G C C C T G G A G G A A A A G \ldots$ In the sequence $s$, there is a $X$ motif $m_{1}=$ GGTGCCGAAGCCCTGGAGGAA of cardinal $c=5$ trinucleotides $\{C T G, G A A, G A G, G C C, G G T\}$ and length $l=7$ trinucleotides. Note that $m_{1}$ cannot be extended to the left or to the right in $s$ due to the presence of the periodic trinucleotide $A A A$ (left) and the trinucleotide $A A G$ (right) which both do not belong to the circular code $X$. Then, the reading frame of the sequence $s$ can easily be deduced from the $X$ motif $m_{1}: \ldots, A A A, G G T, G C C, G A A, G C C, C T G, G A G, G A A, A A G, \ldots$

Definition 7. For simplification reasons, a non- $X$ motif $m(\bar{X})$ is any word of any cardinality and length constructed from the nucleotide 4-letter alphabet $B=\{A, C, G, T\}$ except the $X$ motifs $m(X)$ defined in 
Definition 6. As for the $X$ motifs $m(X)$, we only consider the non- $X$ motifs $m(\bar{X})$ found in reading frame of genes.

Note that using this simplified notation, the class of motifs of $X$ of cardinality $c<4$ trinucleotides and length $l<4$ trinucleotides belong to the non- $X$ motifs $m(\bar{X})$.

In order to evaluate the statistical significance of $X$ motifs in genes, we define, as in Michel et al. (2017), random motifs.

Definition 8. A $R$ random motif $m(R)$ constructed from a random code $R$, is a word with cardinality $4 \leq$ $c \leq 20$ trinucleotides and length $l \geq c \geq 4$ trinucleotides. A random code $R$ is generated according to the properties of $X$, except its circularity property:

(i) $R$ has a cardinality equal to 20 trinucleotides;

(ii) The total number of each nucleotide $A, C, G$ and $T$ in $R$ is equal to 15 (note that $20 \times 3=15 \times 4$ );

(iii) $R$ has no stop trinucleotides $\{T A A, T A G, T G A\}$ and no periodic trinucleotides $\{A A A, C C C, G G G, T T T\}$;

(iv) $R$ is not a circular code. Its associated graph $\mathcal{G}(R)$ is cyclic $(\mathcal{G}(R)$ being not shown).

As for the $X$ motifs $m(X)$ and the non- $X$ motifs $m(\bar{X})$, we only consider the $R$ random motifs $m(R)$ found in reading frame of genes.

In order to obtain a statistically significant distribution of random codes, a set of 100 (different) random codes $R$ are generated according to Definition 8. Examples of such random codes are given in Appendix in Michel et al. (2017).

Definition 9. We say that a letter $N_{i} \in B$ belongs to a motif $m$ of length $l(m)$ if $1 \leq i \leq l(m)$.

Notation 2. $\mathcal{S}=\{X, \bar{X}, R\}$ denotes the three trinucleotide codes associated with the studied motifs $m(X), m(\bar{X})$ and $m(R)$.

\subsection{Multiple alignment of genes}

In the following sections, we briefly recall the multiple alignment of genes and the notations used. A reference gene sequence $s_{1}=\mathbb{R}$ (by convention here the reference sequence is the first sequence in the alignment) is aligned with its orthologous corresponding $n-1$ genes $s_{2}, \ldots, s_{n}$ where $s_{2}, \ldots, s_{n} \in B^{+}$. The genes $s_{1}, s_{2}, \ldots, s_{n}$ have respective lengths $\left|s_{1}\right|,\left|s_{2}\right|, \ldots,\left|s_{n}\right|$. Note that orthologous genes originate from a common DNA ancestral sequence and diverged after a speciation event.

A gene multiple alignment $s_{1}, s_{2}, \ldots, s_{n}, n \geq 2$, is a mapping $z$ on the alphabet $(B \cup\{\varepsilon\})^{n} \backslash(\{\varepsilon\})^{n}$ whose projection on the 1 st component is $s_{1}$, up to the projection on the $n$th component is $s_{n}$. Thus, a gene multiple alignment $z$ of letter length $l$ is noted

$$
z=\left(\begin{array}{ccc}
\bar{N}_{11} & \cdots & \bar{N}_{l 1} \\
\bar{N}_{12} & \cdots & \bar{N}_{l 2} \\
\vdots & \vdots & \vdots \\
\bar{N}_{1 n} & \cdots & \bar{N}_{l n}
\end{array}\right)
$$

with the reference sequence $\mathbb{R}=s_{1}=\bar{N}_{11}, \cdots, \bar{N}_{l 1}$, up to the sequence $s_{n}=\bar{N}_{1 n}, \cdots, \bar{N}_{l n}$ such that the nucleotide $\bar{N}_{j i} \in B \cup\{\varepsilon\}$ for $i=1, \ldots, n$ and $j=1, \ldots, l$ and, where $\varepsilon$ being classically associated with the gap symbol "-" or ".". An aligned tuple $\left(\bar{N}_{j 1}, \ldots, \bar{N}_{j i}, \ldots, \bar{N}_{j n}\right)$ at the $j$ th position such that $\bar{N}_{j 1}, \bar{N}_{j i} \in B$ with 
$\bar{N}_{j 1} \neq \bar{N}_{j i}$ and $i \geq 2$ denotes the substitution of the $j$ th nucleotide $\bar{N}_{j 1}$ of $\mathbb{R}$ by the $j$ th nucleotide $\bar{N}_{j i}$ of $s_{i}$. An aligned tuple $\left(\bar{N}_{j 1}, \ldots, \bar{N}_{j i}, \ldots, \bar{N}_{j n}\right)$ such that $\bar{N}_{j 1} \in B$ and $\bar{N}_{j i} \in\{\varepsilon\}$ with $i \geq 2$ denotes the deletion of the $j$ th nucleotide $\bar{N}_{j 1}$ of $\mathbb{R}$. An aligned tuple $\left(\bar{N}_{j 1}, \ldots, \bar{N}_{j i}, \ldots, \bar{N}_{j n}\right)$ such that $\bar{N}_{j 1} \in\{\varepsilon\}$ and $\bar{N}_{j i} \in B$ with $i \geq$ 2 denotes the insertion of the $j$ th nucleotide $\bar{N}_{j i}$ of $s_{i}$.

The $X$ motifs $m(X)$, the non- $X$ motifs $m(\bar{X})$ and the $R$ random motifs $m(R)$ located in the gene multiple alignment belong to the alphabet $B \cup\{\varepsilon\}$, i.e. they may contain gaps. Those which are located in the gene sequence $s_{i}, i=1, \ldots, n$, are noted $m\left(X, s_{i}\right), m\left(\bar{X}, s_{i}\right)$ and $m\left(R, s_{i}\right)$, respectively, in particular, $m(X, \mathbb{R})$, $m(\bar{X}, \mathbb{R})$ and $m(R, \mathbb{R})$, respectively, in the reference gene $\mathbb{R}$. Figure 1 shows an example of a part of a gene multiple alignment.

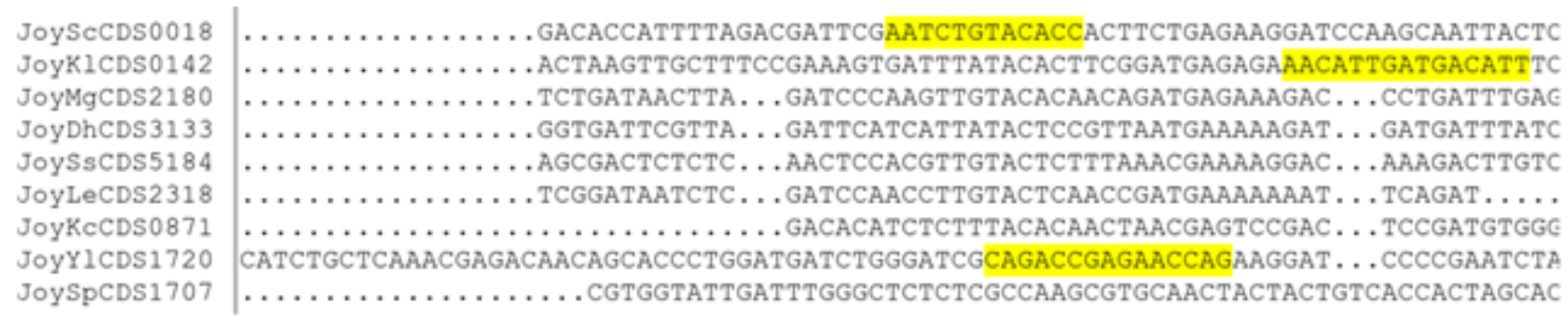

No.Xmotifs 00000000000000000000000000000000000000011111122222211111111100011111111111111100

Figure 1. Screenshot of a yeast gene multiple alignment. The $X$ motifs $m(X)$ in the reading frame of genes (Definition 6: cardinality $4 \leq c \leq 20$ trinucleotides and length $l \geq c \geq 4$ trinucleotides) are coloured in yellow. The reference (first) gene $s_{1}=\mathbb{C}$ (Saccharomyces Cerevisiae $S c$ ) contains one $X$ motif $m(X, \mathbb{C})$ in reading frame and two non- $X$ motifs $m(\bar{X}, \mathbb{C})$ in reading frame (Definition 7 ) without colour. Two $X$ motifs are identified in the reading frame of two other yeast genes: $m(X, \mathbb{L})$ (Kluyveromyces lactis Kl, $s_{2}=\mathbb{L}$ ) and $m\left(X, s_{8}\right)$ (Yarrowia lipolyticai $Y I$ ). The number of $X$ motifs (last row) is used in the calculation of the positional conservation parameter (Section 2.4).

\subsection{Positional conservation parameter of $X$ motifs and random motifs}

Here, we consider whether the position of $X$ motifs is preserved within the genes from different organisms. To do this, for each column of a gene alignment, the number of organisms with a $X$ motif at this position was calculated. For example, in Figure 1, the number of organisms with a $X$ motif is equal to 0,1 , or 2 . This number was normalized by the number of organisms having a nucleotide at that position in the alignment and not a gap.

Formally, we define a simple statistical parameter for analysing the positional conservation of motifs in the reference genes in the multiple alignments.

Definition 10. The positional conservation score $\operatorname{Ppc}(m)$ of all motifs $m=(m(\mathcal{S}, \mathbb{R})), \mathcal{S} \in\{X, R\}$ for studying $X$ motifs and $R$ random motifs, of letter lengths $l(m), m$ on the alphabet $B \cup\{\varepsilon\}$ (with gaps), in the reference genes $s_{1}=\mathbb{R}$ in all the gene multiple alignments $s_{1}, s_{2}, \ldots, s_{n}$ is equal to

$$
\operatorname{Ppc}(m)=\operatorname{Ppc}(m(\mathcal{S}, \mathbb{R}))=\frac{1}{\sum_{m \in \mathbb{R}} l(m)} \sum_{m \in \mathbb{R}} \sum_{j=1}^{l(m)} \frac{1}{N b_{j}} \sum_{i=1}^{n} \delta_{i, j}
$$

where 


$$
\delta_{i, j}=\left\{\begin{array}{c}
1 \text { if } N_{j i} \in B \text { and } N_{j i} \in m\left(\mathcal{S}, s_{i}\right) \\
0 \text { otherwise }
\end{array}\right.
$$

$N b_{j}$ is the number of nucleotides without gaps at position $j$ in the multiple alignment of $n$ genes, $2 \leq$ $N b_{j} \leq n$ for $j=1, \ldots, l(m)$. The condition $N_{j i} \in B$ and $N_{j i} \in m\left(\mathcal{S}, s_{i}\right)$ signifies that the letter $N_{j i}$ at the $j t h$ position in the gene $s_{i}$ is a nucleotide and not a gap, and belongs to a motif $m$.

Remark 1. The positional score $P p c(m) \in] 0,1]$. The positional conservation $P p c$ of the motif $m$ is the lowest in the alignment when $P p c(m) \approx 0$, i.e. when the motif $m$ in the reference genome is aligned with zero motifs in the other genomes. The positional conservation $P p c$ of the motif $m$ is the highest in the alignment when $\operatorname{Ppc}(m)=1$ corresponding to the case where all genes without gaps have $X$ motifs in the same position as the reference genes.

\subsection{Pairwise alignment parameters of $X$ motifs and non- $X$ motifs}

A pairwise alignment is a multiple alignment $z$ with $n=2$ sequences of letter length $l$ such that their nucleotides $N \in B \cup\{\varepsilon\}$ (with gaps). Several classical pairwise alignment parameters are used to estimate the conservation of a pairwise alignment, including (i) the percentage of alignment positions that contain identical nucleotides, and (ii) the ratio of synonymous to non-synonymous substitutions. These parameters are briefly recalled in the following definitions and are illustrated with examples from Figure 2 , and Table 1 and Table 2.

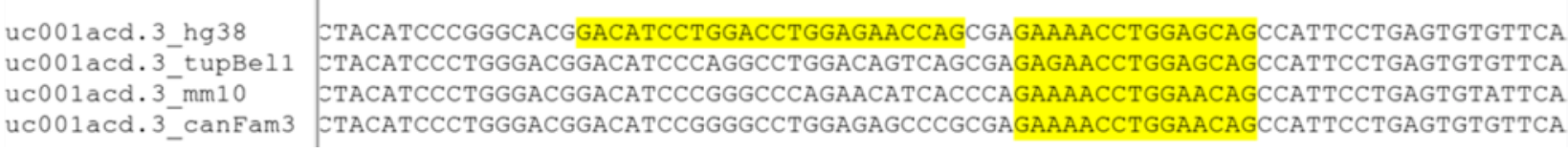

Figure 2. Screenshot of a mammal gene multiple alignment. The $X$ motifs $m(X)$ in the reading frame of genes (Definition 6: cardinality $4 \leq c \leq 20$ trinucleotides and length $l \geq c \geq 4$ trinucleotides) are coloured in yellow. The reference (first) gene $s_{1}=\mathbb{H}$ (Homo sapiens hg38) contains two $X$ motifs $m(X, \mathbb{H})$ in reading frame and three non- $X$ motifs $m(\bar{X}, \mathbb{H})$ in reading frame (Definition 7) without colour. The 2 nd non- $X$ motif $m(\bar{X}, \mathbb{H})$ is composed of one trinucleotide $C G A \notin X(1)$. Three $X$ motifs are identified in the reading frame of three other mammal genes: $m\left(X, s_{2}\right)$ (Tupaia Belangeri tupBel1), $m(X, \mathbb{M})$ (Mus musculus $m m 10, s_{3}=\mathbb{M}$ ) and $m\left(X, s_{4}\right)$ (Canis Lupus Familiaris canFam3).

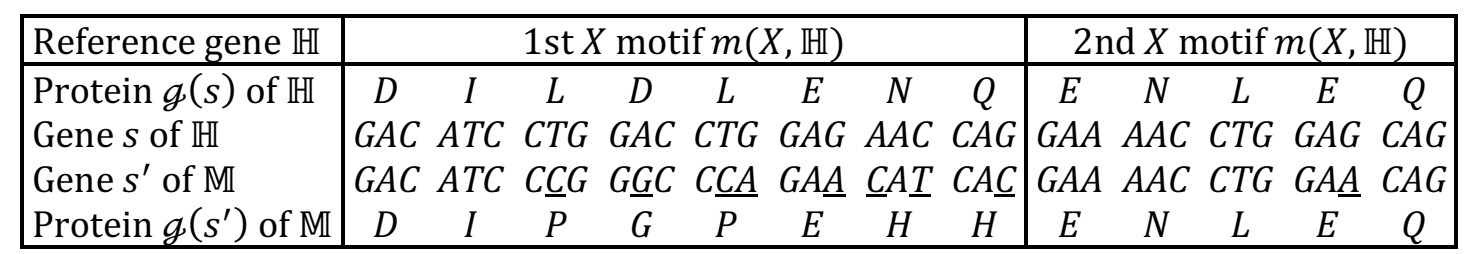

Table 1. From Figure 2 , the alignment of the two $X$ motifs $m(X, \mathbb{H})$ in reading frame of total length 39 nucleotides of the reference gene $s_{1}=\mathbb{H}$ (Homo sapiens hg38) and the gene $\mathbb{M}$ (Mus musculus mm10). There are 30 identical nucleotide pairs and 9 different nucleotide pairs (underlined). The protein alignment associated with the gene alignment is given by applying the universal genetic code map $g$ (Definition 3) to each trinucleotide. 


\begin{tabular}{|c|c|c|c|}
\hline Reference gene $\mathbb{H}$ & 1st non- $X$ motif $m(\bar{X}, \mathbb{H})$ & 2nd $m(\bar{X}, \mathbb{H})$ & 3rd non- $X$ motif $m(\bar{X}, \mathbb{H})$ \\
\hline$g(s)$ of $\mathbb{H}$ & $P \quad G$ & $\vec{R}$ & $\begin{array}{lll}P & F & L\end{array}$ \\
\hline Gen & TAC ATC CCG GGC ACG & $C G A$ & CCA TTC CTG AGT GTG TTC \\
\hline of $\mathbb{M}$ & TAC ATC CCT GGG ACG & $C \underline{C A}$ & CCA TTC CTG AGT GTA TTC \\
\hline Protein $q\left(s^{\prime}\right)$ of $\mathbb{M}$ & $P$ & $\bar{P}$ & $F$ \\
\hline
\end{tabular}

Table 2. From Figure 2 , the alignment of the three non- $X$ motifs $m(\bar{X}, \mathbb{H})$ in reading frame of total length 36 nucleotides of the reference gene $s_{1}=\mathbb{H}$ (Homo sapiens $\mathrm{hg} 38$ ) and the gene $\mathbb{M}$ (Mus musculus mm10). There are 32 identical nucleotide pairs and 4 different nucleotide pairs (underlined). The protein alignment associated with the gene alignment is given by applying the universal genetic code map $g$ (Definition 3) to each trinucleotide.

Definition 11. The percentage $\operatorname{Pid}(m)$ of identical nucleotides of all motifs $m=(m(\mathcal{S}, \mathbb{R})), \mathcal{S} \in\{X, \bar{X}\}$ for studying $X$ motifs and non- $X$ motifs, of letter lengths $l(m), m$ on the alphabet $B \cup\{\varepsilon\}$ (with gaps), in the reference genes $s_{1}=\mathbb{R}$ in all the gene pairwise alignments $s_{1}$ and $s_{2}$ is equal to

$$
\operatorname{Pid}(m)=\operatorname{Pid}(m(\delta, \mathbb{R}))=\frac{1}{\sum_{m \in \mathbb{R}} l(m)} \sum_{m \in \mathbb{R}} \sum_{i=1}^{l(m)} \delta_{i}
$$

where the operator $\delta_{i}, 1 \leq i \leq l(m)$, associated with a pair of letters $N$ is defined by

$$
\delta_{i}=\left\{\begin{array}{c}
1 \text { if } N_{i 1} \in B \text { and } N_{i 1}=N_{i 2} . \\
0 \text { otherwise }
\end{array}\right.
$$

Example 3. From Table $1, \operatorname{Pid}(m(X, \mathbb{H}))=30 / 39=76.92 \%$.

Definition 12. Let $f_{i}(c), g_{i}(c)$ respectively, be the fraction of synonymous, non-synonymous respectively, potential substitutions at the $i$ th site, $i=1,2,3$, of a given codon $c=N_{1} N_{2} N_{3}$. Then, the numbers $N s(c), N n s(c)$ respectively, of synonymous, non-synonymous respectively, sites for a given codon $c$, are defined according to Nei and Gojobori (1986) by $N s(c)=\sum_{i=1}^{3} f_{i}(c)$ and $N n s(c)=$ $\sum_{i=1}^{3} g_{i}(c)=\sum_{i=1}^{3}\left(1-f_{i}(c)\right)=3-N s(c)$.

Example 4. In the case of the codon $c=C T G$ coding the amino acid $g(c)=L e u, f_{1}($ Leu $)=\frac{1}{3}$ as only the 1st site substitution $C T G \rightarrow T T G$ is synonymous out of $A T G, G T G$ and $T T G, f_{2}(L e u)=0$ as there is no 2nd site synonymous substitution out of $C A G, C C G$ and $C G G$, and $f_{3}(L e u)=\frac{3}{3}=1$ as all the 3rd site substitutions are synonymous out of CTA, CTC and CTT. Then, $N s($ Leu $)=\frac{1}{3}+0+1=\frac{4}{3}$ and $N n s($ Leu $)=$ $3-\frac{4}{3}=\frac{5}{3}$.

The definitions of $N s(c)$ and $N n s(c)$ for a given codon are naturally extended to a motif $m$.

Definition 13. The potential numbers $N s(m), N n s(m)$ respectively, of synonymous, non-synonymous respectively, sites for a motif $m=(m(\mathcal{S}, \mathbb{R})), \mathcal{S} \in\{X, \bar{X}\}$ for studying $X$ motifs and non- $X$ motifs, of letter length $l, m$ on the alphabet $B$ (without gaps), are equal to $N s(m)=\sum_{c \in m} N s(c)$ where $N s(c)$ is defined in Definition 12, and $N n s(m)=l-N s(m)$. Then, $N s(m)$ and $N n s(m)$ are computed for all motifs $m$ in the reference sequence $s_{1}$ of the gene pairwise alignments. 
Example 5. From Table 1 , the potential numbers $N s(m)$ and $N n s(m)$ of synonymous and nonsynonymous sites for the two $X$ motifs are $N s(m(X, \mathbb{H}))=\frac{1}{3}+\frac{2}{3}+\frac{4}{3}+\frac{1}{3}+\frac{4}{3}+\frac{1}{3}+\frac{1}{3}+\frac{1}{3}+\frac{1}{3}+\frac{1}{3}+\frac{4}{3}+$ $\frac{1}{3}+\frac{1}{3}=\frac{23}{3} \approx 7.67$ and $N n s(m(X, \mathbb{H}))=39-\frac{23}{3}=\frac{94}{3} \approx 31.33$.

Definition 14. Let $O s(m)$, Ons $(m)$ respectively, be the observed numbers of synonymous, nonsynonymous respectively, substitutions of a reference motif $m=(m(\mathcal{S}, \mathbb{R})), \mathcal{S} \in\{X, \bar{X}\}$ in the gene $s_{1}=$ $\mathbb{R}$ for studying $X$ motifs and non- $X$ motifs, in the motif $m^{\prime}$ in gene $s_{2}$ of letter lengths $l(m)=l\left(m^{\prime}\right), m, m^{\prime}$ on the alphabet $B$ (without gaps), in all gene pairwise alignments $s_{1}$ and $s_{2}$.

Remark 2. Os $(m)+O n s(m)=l(m)-\sum_{i=1}^{l(m)} \delta_{i}$ where $\delta_{i}$ is defined in Definition 11.

Example 6. From Table $1, O s(m(X, \mathbb{H}))=4$ (four synonymous substitutions: the 3rd site of $C T G(L)$, $G A G(E) \rightarrow G A A(E)$, the 3rd site of $A A C(N)$ and $G A G(E) \rightarrow G A A(E))$ and $\operatorname{Ons}(m(X, \mathbb{H}))=5$ (five non-synonymous substitutions: $C T G(L) \rightarrow C C G(P), \quad G A C(D) \rightarrow G G C(G), \quad C T G(L) \rightarrow C C A(P)$, $A A C(N) \rightarrow C A T(H)$ and $C A G(Q) \rightarrow C A C(H))$.

Definition 15. The percentages $\operatorname{Ps}(m), \operatorname{Pns}(m)$ respectively, of synonymous, non-synonymous respectively, substitutions of a reference motif $m=(m(\mathcal{S}, \mathbb{R})), \mathcal{S} \in\{X, \bar{X}\}$ in the gene $s_{1}=\mathbb{R}$ for studying $X$ motifs and non- $X$ motifs, in the motif $m^{\prime}$ in the gene $s_{2}$ of letter lengths $l(m)=l\left(m^{\prime}\right), m, m^{\prime}$ on the alphabet $B$ (without gaps), in all gene pairwise alignments $s_{1}$ and $s_{2}$, are equal to $P s(m)=$ $O s(m) / N s(m)$ and $P n s(m)=O n s(m) / N n s(m)$ where $O s(m)$ and $O n s(m)$ are defined in Definition 14, and $N s(m)$ and $N n s(m)$ in Definition 13.

Example 7. From Table 1, $P s(m(X, \mathbb{H}))=\frac{4}{\frac{23}{3}}=\frac{12}{23} \approx 0.52$ and $P n s(m(X, \mathbb{H}))=\frac{5}{\frac{94}{3}}=\frac{15}{94} \approx 0.16$.

Example 8 summarizes the parameters for the three non- $X$ motifs of Table 2.

Example 8. From Table 2 $\operatorname{Pid}(m(\bar{X}, \mathbb{H}))=32 / 36=88.89 \%, N s(m(\bar{X}, \mathbb{H}))=\frac{1}{3}+\frac{2}{3}+\frac{3}{3}+\frac{3}{3}+\frac{3}{3}+\frac{4}{3}+$ $\frac{3}{3}+\frac{1}{3}+\frac{4}{3}+\frac{1}{3}+\frac{3}{3}+\frac{1}{3}=\frac{29}{3} \approx 9.67, \operatorname{Nns}(m(\bar{X}, \mathbb{H}))=3 \times 12-\frac{29}{3}=\frac{79}{3} \approx 26.33, \operatorname{Os}(m(\bar{X}, \mathbb{H}))=3($ three synonymous substitutions: $C C G(P) \rightarrow C C T(P), G G C(G) \rightarrow G G G(G)$ and $G T G(V) \rightarrow G T A(V)$ and $\operatorname{Ons}(m(\bar{X}, \mathbb{H}))=1$ (one non-synonymous substitution: $C G A(R) \rightarrow C C A(P)), \operatorname{Ps}(m(\bar{X}, \mathbb{H}))=\frac{3}{\frac{29}{3}}=$ $\frac{9}{29} \approx 0.31$ and $\operatorname{Pns}(m(\bar{X}, \mathbb{H}))=\frac{1}{\frac{79}{3}}=\frac{3}{79} \approx 0.04$.

\subsection{Codon substitution matrix of $X$ motifs and random motifs}

We define a codon (trinucleotide) substitution matrix $\mathbf{A}(m(\mathcal{S}, \mathbb{R})), \mathcal{S} \in\{X, R\}$ for studying $X$ motifs and $R$ random motifs, in the reference genes $s_{1}=\mathbb{R}$ in all the gene multiple alignments $s_{1}, s_{2}, \ldots, s_{n}$. The codon substitution matrix $\mathbf{A}(m(\mathcal{S}, \mathbb{R}))=\left[a_{i j}\right]_{1 \leq i \leq 64,1 \leq j \leq 64}$ of size $64 \times 64$ (square matrix) where the 64 rows and the 64 columns are associated with the 64 codons $B^{3}$, has element $a_{i j}=N b\left(\mathcal{S}[j] \rightarrow B^{3}[i]\right)$ in row $i$ and column $j$ referring to the number of substitutions of codon $\mathcal{S}[j]$ (jth codon of $\mathcal{S}$ ) of the motifs $m$ (in the reference genes $\mathbb{R}$ ) by the aligned codon $B^{3}[i]$ (ith codon of $B^{3}$ ) of the $n-1$ genes $s_{2}, \ldots, s_{n}$. 
We define the normalized matrix $\mathbf{B}(m(\mathcal{S}, \mathbb{R}))=\left[b_{i j}\right]_{1 \leq i \leq 64,1 \leq j \leq 64}$ with element $b_{i j}=a_{i j} / a_{. j}$ where $a_{. j}=$ $\sum_{k=1}^{64} a_{k j}\left(a_{. j} \neq 0\right)$, for $1 \leq i \leq 64$ and $1 \leq j \leq 64$, such that it is stochastic in column. The column normalization, rather than a full matrix normalization, allows the codons to be compared whatever the codon usage.

Remark 3. The elements $a_{i i}$ of $\mathbf{A}$ and $b_{i i}$ of $\mathbf{B}$ can be indexed either by numbers or by the codons $B^{3}$.

Remark 4. The diagonal elements $a_{i i}$ of $\mathbf{A}$ and $b_{i i}$ of $\mathbf{B}$ can be different from 0 .

Remark 5. For a given code $\mathcal{S}$, i.e. the circular code $X$ or a given random code $R$, with $\operatorname{Card}(\mathcal{S})=20$ codons $\mathcal{S}$ (Section 2.2), the matrices $\mathbf{A}$ and $\mathbf{B}$ have 20 non-empty codon columns and $64-20=44$ empty codon columns. However, the 20 codon columns of $\mathbf{A}$ and $\mathbf{B}$ vary with each (different) random code $R$, which obviously differ from the 20 codon columns of the circular code $X$.

Example 9. An example of construction of the matrices $\mathbf{A}$ and $\mathbf{B}$ is given from the alignment in Table 3. The first codon column leads to the submatrix A given in Table 4. The procedure is iterated for each codon column and leads to the matrix A given in Table 5. The normalized matrix $\mathbf{B}$ is given in Table 6.

\begin{tabular}{|l|llllllll|}
\hline$s_{1}$ & $G A G$ & $G A C$ & $A T C$ & $C T G$ & $G A C$ & $C T G$ & $A A C$ & $C A G$ \\
$s_{2}$ & $G A C$ & $G A C$ & $A T C$ & $C C A$ & $G G C$ & $C T G$ & $A G T$ & $C A G$ \\
$s_{3}$ & $G A A$ & $G A C$ & $A T C$ & $C C G$ & $G G C$ & $C C A$ & $C A T$ & $C A C$ \\
$s_{4}$ & $G A G$ & $G A C$ & $A T C$ & $C G G$ & $G G C$ & $C T G$ & $A G C$ & $C C G$ \\
\hline
\end{tabular}

Table 3. Example of a multiple alignment of four genes where $s_{1}=\mathbb{R}$ is the reference gene.

\begin{tabular}{c|cl}
$\mathbf{A}$ & $G A G$ & \\
\hline$G A A$ & 1 & $G A G \rightarrow G A A$ \\
$G A C$ & 1 & $G A G \rightarrow G A C$ \\
$G A G$ & 1 & $G A G \rightarrow G A G$ \\
\hline
\end{tabular}

Table 4. Codon substitution submatrix $\mathbf{A}$ of the first codon column from example of Table 3.

\begin{tabular}{c|cccccc} 
A & $A A C$ & $A T C$ & $C A G$ & $C T G$ & $G A C$ & $G A G$ \\
\hline$A G C$ & 1 & & & & & \\
$A G T$ & 1 & & & & & \\
$A T C$ & & 3 & & & & \\
$C A C$ & & & 1 & & & \\
$C A G$ & & & 1 & & & \\
$C A T$ & 1 & & & & & \\
$C C A$ & & & & 2 & & \\
$C C G$ & & & 1 & 1 & & \\
$C G G$ & & & & 1 & & \\
$C T G$ & & & & 2 & & \\
$G A A$ & & & & & & 1 \\
$G A C$ & & & & & 3 & 1 \\
$G A G$ & & & & & 3 & 1 \\
$G G C$ & & & & & 3
\end{tabular}

Table 5. Codon substitution matrix A from example of Table 3. The remaining codon rows and columns equal to 0 are not shown. 


\begin{tabular}{c|cccccc}
$\mathbf{B}$ & $A A C$ & $A T C$ & $C A G$ & $C T G$ & $G A C$ & $G A G$ \\
\hline$A G C$ & $1 / 3$ & & & & & \\
$A G T$ & $1 / 3$ & & & & & \\
$A T C$ & & 1 & & & & \\
$C A C$ & & & $1 / 3$ & & & \\
$C A G$ & & & $1 / 3$ & & & \\
$C A T$ & $1 / 3$ & & & & & \\
$C C A$ & & & & $1 / 3$ & & \\
$C C G$ & & & $1 / 3$ & $1 / 6$ & & \\
$C G G$ & & & & $1 / 6$ & & \\
$C T G$ & & & & $1 / 3$ & & \\
$G A A$ & & & & & & $1 / 3$ \\
$G A C$ & & & & & $1 / 2$ & $1 / 3$ \\
$G A G$ & & & & & $1 / 2$ & $1 / 3$ \\
$G G C$ & & & & & $1 / 2$ & \\
\hline Sum & 1 & 1 & 1 & 1 & 1 & 1
\end{tabular}

Table 6. Normalized matrix $\mathbf{B}$ from example of Table 3 . The remaining codon rows and columns equal to 0 are not shown.

\subsection{Amino acid conservation parameter of $X$ motifs and random motifs}

We define a simple statistical parameter for analysing the conservation of $X$ motifs and $R$ random motifs for the 12 amino acids $X$ (2) coded by the circular code $X$, in the reference genes $s_{1}=\mathbb{R}$ in all the gene multiple alignments $s_{1}, s_{2}, \ldots, s_{n}$.

Definition 16. The percentage $\operatorname{Paac}(m(\mathcal{S}, \mathbb{R}), p)$ of conservation of $X$ codons per amino acid $p$ (peptide component) coded by all the motifs $m=(m(\mathcal{S}, \mathbb{R})), \mathcal{S} \in\{X, R\}$ for studying $X$ motifs and $R$ random motifs, in the reference genes $s_{1}=\mathbb{R}$ in all the gene multiple alignments $s_{1}, s_{2}, \ldots, s_{n}$, is equal to

$$
\operatorname{Paac}(m(\mathcal{S}, \mathbb{R}), p)=\frac{1}{\operatorname{Card}\left(g^{-1}(p) \cap \mathcal{S}\right)} \sum_{\substack{i, j \in \mathcal{g}^{-1}(p) \\ i, j \in \mathcal{S}}} b_{i j}(m(\mathcal{S}, \mathbb{R}))
$$

where $p \in \mathcal{X}=\{A, D, E, F, G, I, L, N, Q, T, V, Y\}(2), b_{i j}(m(\mathcal{S}, \mathbb{R}))$ is the element of the normalized matrix B of $\mathcal{S}$ defined in Section 2.6 and the inverse genetic code map $g^{-1}$ defined in Definition 3.

Definition 17. The mean percentage $\bar{P} \operatorname{aac}(m(\mathcal{S}, \mathbb{R}), \mathcal{X})$ of conservation of $X$ codons in the 12 amino acids $\mathcal{X}(2)$ coded by all the motifs $m=(m(\mathcal{S}, \mathbb{R})), \mathcal{S} \in\{X, R\}$, in the reference genes $s_{1}=\mathbb{R}$ in all the gene multiple alignments $s_{1}, s_{2}, \ldots, s_{n}$, is equal to

$$
\bar{P} \operatorname{aac}(m(\mathcal{S}, \mathbb{R}), \mathcal{X})=\frac{1}{\operatorname{Card}(\mathcal{X})} \sum_{p \in \mathcal{X}} \operatorname{Paac}(m(\mathcal{S}, \mathbb{R}), p)
$$

where $p \in \mathcal{X}=\{A, D, E, F, G, I, L, N, Q, T, V, Y\}(2)$ and $\operatorname{Paac}(m(\mathcal{S}, \mathbb{R}), p)$ defined in Definition 16.

Remark 6. The mean percentage $\bar{P} a a c$ give the same statistical weight for each amino acid.

Definition 18. To achieve a strong statistical significance, we use the information from the 100 random codes $R\left(R_{1}, \ldots, R_{100}\right)$, and not only one random code, the percentage Paac $(\bar{m}(R, \mathbb{R}), p)$ of conservation of $X$ codons per amino acid $p$ coded by the $R$ mean random motifs $\bar{m}(R, \mathbb{R})$ in the reference genes $s_{1}=$ $\mathbb{R}$ in all the gene multiple alignments $s_{1}, s_{2}, \ldots, s_{n}$, is equal to 


$$
\operatorname{Paac}(\bar{m}(R, \mathbb{R}), p)=\frac{1}{\sum_{k=1}^{100} \delta_{k}} \sum_{k=1}^{100} \operatorname{Paac}\left(m\left(R_{k}, \mathbb{R}\right), p\right)
$$

where $p \in \mathcal{X}=\{A, D, E, F, G, I, L, N, Q, T, V, Y\}(2), \operatorname{Paac}\left(m\left(R_{k}, \mathbb{R}\right), p\right)$ defined in Definition 16 and $\delta_{k}=$ 1 if $g^{-1}(p) \cap R_{k} \neq \varnothing$ (i.e. the random code $R_{k}$ can code the amino acid $p$ ) and $\delta_{k}=0$ otherwise.

Remark 7. For the $R$ mean random motifs $\bar{m}(R, \mathbb{R})$, we only analyse in the mean matrix $\overline{\mathbf{B}}$ the trinucleotides coding the 12 amino acids $X$ of the circular code $X$.

Definition 19. The mean percentage $\bar{P} a a c(\bar{m}(R, \mathbb{R}), X)$ of conservation of $X$ codons in the 12 amino acids $\mathcal{X}$ (2) coded by the $R$ mean random motifs $\bar{m}(R, \mathbb{R})$ in the reference genes $s_{1}=\mathbb{R}$ in all the gene multiple alignments $s_{1}, s_{2}, \ldots, s_{n}$, is equal to

$$
\overline{\operatorname{Paac}}(\bar{m}(R, \mathbb{R}), \mathcal{X})=\frac{1}{\operatorname{Card}(\mathcal{X})} \sum_{p \in \mathcal{X}} \operatorname{Paac}(\bar{m}(R, \mathbb{R}), p)
$$

where $p \in \mathcal{X}=\{A, D, E, F, G, I, L, N, Q, T, V, Y\}(2)$ and $\operatorname{Paac}(\bar{m}(R, \mathbb{R}), p)$ defined in Definition 18.

\subsection{Data}

In order to increase the significance of the results, two classes of independent alignments are investigated. The first class of alignments is based on four mammal genomes, which represent highly evolved species and closely related genomes. The second class of alignments is built from nine yeast genomes, which represent the simplest eukaryotes and are more divergent. The human genome for the first class and the Saccharomyces cerevisiae genome for the second class are taken as reference genomes as they are well documented model organisms.

\subsubsection{Mammal gene alignments}

From the mammalian gene multiple alignments available on the UCSC site (https://bds.mpicbg.de/hillerlab/ 144VertebrateAlignment_CESAR/, Sharma and Hiller, 2017), we have used genes from four well annotated genomes. Table 7 shows some summary statistics of the four selected mammal genomes.

\begin{tabular}{|llcc|}
\hline Genome name & Identification & $\begin{array}{c}\text { Number } \\
\text { of genes }\end{array}$ & $\begin{array}{c}\text { Nucleotide length } \\
\text { of genes }\end{array}$ \\
\hline Canis lupus familiaris & canFam3 & 21,137 & $34,379,490$ \\
Homo sapiens & hg38 $(\mathbb{H})$ & 22,352 & $36,808,167$ \\
Mus musculus & mm10 $(\mathbb{M})$ & 20,178 & $33,519,381$ \\
Tupaia belangeri & tupBel1 & 18,485 & $23,387,559$ \\
\hline
\end{tabular}

Table 7. Genomes of four mammals.

H. sapiens $(\mathrm{hg} 38, \mathbb{H})$ is taken as the reference genome and is present in each of the 22,352 gene alignments. One or two corresponding genes from the three other species may be missing, in which case the corresponding genes are replaced by gaps in the alignment. 


\subsubsection{Yeast gene alignments}

For the yeast multiple alignments, the protein sequences of nine different yeasts and the localization of the corresponding nucleic acid sequence on the chromosomes (Table 8) are obtained from the NCBI Genbank (https://www.ncbi.nlm.nih.gov/genbank/).

\begin{tabular}{|llcc|}
\hline Genome name & Identification & $\begin{array}{c}\text { Number } \\
\text { of genes }\end{array}$ & $\begin{array}{c}\text { Nucleotide length } \\
\text { of genes }\end{array}$ \\
\hline Debaryomyces hansenii & Dh & 6288 & $7,506,066$ \\
Kluyveromyces lactis & $K l(\mathbb{L})$ & 5085 & $7,729,998$ \\
Kuraishia capsulata & $K c$ & 5989 & $6,911,424$ \\
Lodderomyces elongisporus & Le & 5799 & $7,110,237$ \\
Meyerozyma guilliermondii & $M g$ & 5920 & $6,633,972$ \\
Saccharomyces cerevisiae & Sc $(\mathbb{C})$ & 6008 & $8,246,529$ \\
Scheffersomyces stipitis & $S s$ & 5818 & $6,991,422$ \\
Schizosaccharomyces pombe & $S p$ & 4980 & $5,614,506$ \\
Yarrowia lipolytica & $Y l$ & 6472 & $6,762,072$ \\
\hline
\end{tabular}

Table 8. Genomes of nine yeasts.

S. cerevisiae $(\mathrm{Sc}, \mathbb{C}$ ) is taken as the reference genome. A BLAST (Altschul et al., 1997) database of all protein sequences of these nine organisms is created. For all protein sequences of $S$. cerevisiae, a BLAST search in this database is performed. Then, the protein alignments containing from 2 to 9 sequences are obtained using ClustalW (Thompson et al., 1994). The corresponding nucleic sequence alignments are created by localizing each amino acid on the genome. The BLAST searches, alignments and some data analyses were performed using our in-house software platform Gscope (R. Ripp, unpublished, details in Section 2.9).

\subsection{Software development}

In a nucleic sequence alignment, the $X$ and $R$ random motifs are localized in the genes using a program developed in the Java language (El Soufi and Michel, 2017). The program takes optional parameters that define the minimum cardinality $c$ (in trinucleotides) and the length $l$ (in trinucleotides) of the $X$ and $R$ motifs searched. The $X$ and $R$ motifs verify Definition 6 (cardinality $c \geq 4$ trinucleotides and with any length $l \geq c \geq 4$ trinucleotides). Although the $X$ and $R$ motifs are contiguous in the gene sequences, gaps may be inserted during the alignment process.

Gscope is an integrated platform allowing the analysis of all kinds of genomic data. It is written in Tcl/Tk and runs under all operating systems. It is specifically designed to perform high throughput analyses. Gscope includes the tools necessary to create the basic data, analysis tools and visualization interfaces. It also allows the creation of SQL relational databases and the querying and display of the available information through a web based interface (Wscope). 


\section{Results}

The results presented below are based on basic frequency statistics and their biological significance is clear. In order to evaluate the statistical significance of the different results presented below, we chose an approach that involved comparing the results obtained for the $X$ motifs with those obtained for $R$ random motifs generated by 100 (different) random codes $R$. This approach avoids the problems associated with defining statistical hypotheses about the nucleotide composition, the length and the random model of the different regions of the genome. The main disadvantage of our approach is the additional computational resources required to obtain the results for the 100 random codes.

This section is divided into two main parts. In the first part, we estimate the evolutionary conservation of $X$ motifs in two large-scale sets of genes from mammal and yeast species ( $\sim 20,000$ and $\sim 6000$ genes, respectively). In the second part, we evaluate the potential functional activity of the $X$ motifs, by correlating them with existing experimental data.

\subsection{Evolutionary conservation of $X$ motifs in mammal and yeast genes}

\subsubsection{Enrichment of $X$ motifs in mammal and yeast genes}

We first investigated the occurrence number and codon length of $X$ motifs in the two sets of genes. Figure 3 and Figure 4 show a very strong enrichment of $X$ motifs in both mammal and yeast genes compared to the $R$ random motifs from the 100 (different) random codes $R$. The number of $X$ motifs in mammal genes is equal to 173,390 , compared to a mean number of $60,330 R$ motifs. This difference is significant according to a one-sided Student's $t$-test with value $p \approx 10^{-82}$. The number of $X$ motifs in yeast genes is equal to 35,833 , compared to a mean number of $15,853 R$ motifs. Again, this difference is significant according to a one-sided Student's $t$-test with value $p \approx 10^{-75}$. This result is an additional and strong confirmation of the enrichment of $X$ motifs in genes previously observed in the yeast $S$. cerevisiae (Michel et al., 2017). 


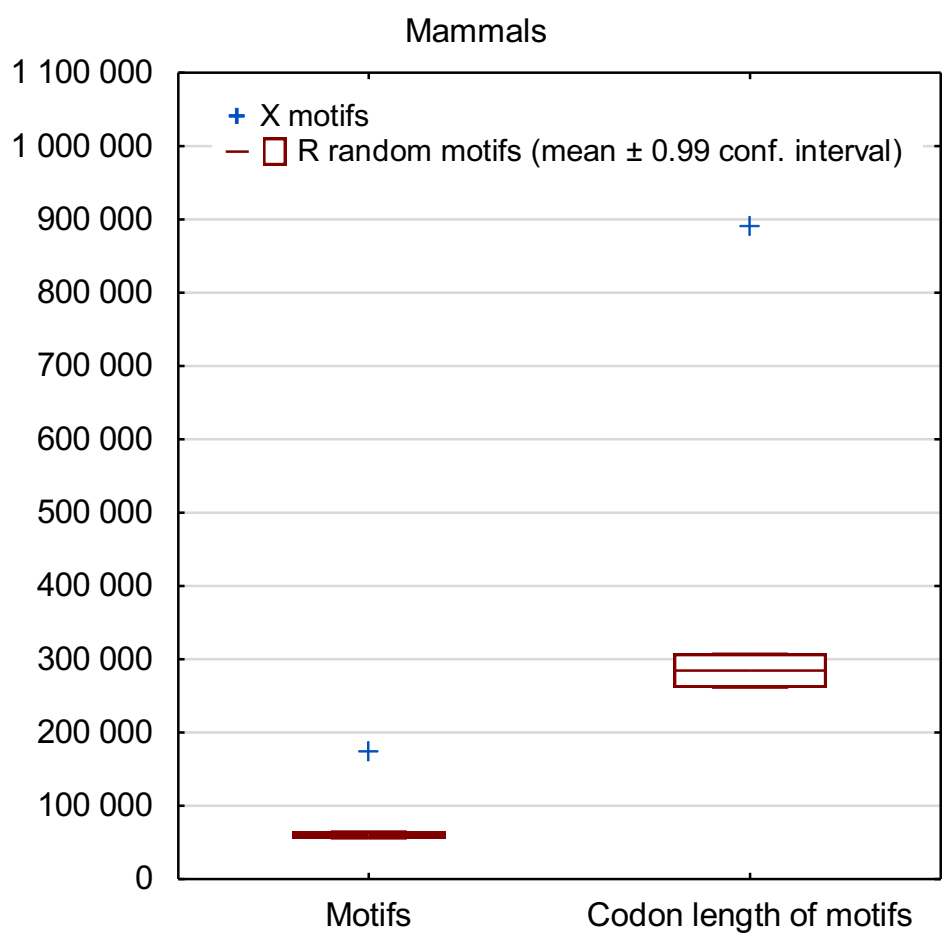

Figure 3. Comparison of the number of $X$ and $R$ random motifs and their codon lengths in mammalian genes. The number of $X$ motifs is represented with a blue cross. The distribution of the $R$ random motifs from the 100 random codes $R$ is indicated by boxplots representing the mean and \pm 0.99 confidence interval.

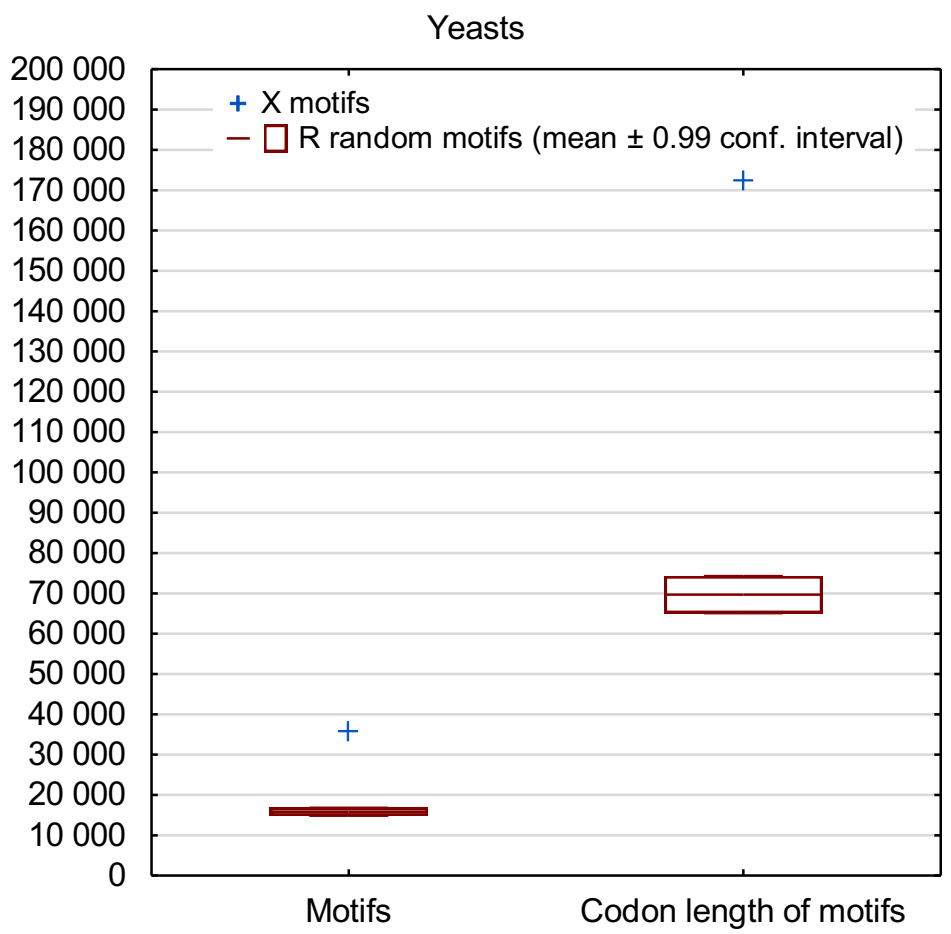

Figure 4. Comparison of the number of $X$ and $R$ random motifs and their codon lengths in the yeast genes. The number of $X$ motifs is represented with a blue cross. The distribution of the $R$ random motifs from the 100 random codes $R$ is indicated by boxplots representing the mean and \pm 0.99 confidence interval. 


\subsubsection{Positional conservation of $X$ motifs in mammal and yeast genes}

We then used the multiple alignments corresponding to the 22,352 mammal genes and the 6008 yeast genes, to calculate the positional conservation scores $P p c(m)$ (Definition 10; $m=(m(\mathcal{S}, \mathbb{R})), \mathcal{S} \in\{X, R\}$ ) for the $X$ motifs and the $R$ random motifs from the 100 (different) random codes $R$, shown in Figure 5 and Figure 6, respectively. The positional conservation score $P p c$ measures the number of motifs that are found in the same columns in a given multiple alignment. For both mammals and yeasts, the number of $X$ motifs with the highest positional conservation score $P p c=1$ was higher than the number of $R$ motifs. In contrast, the number of $X$ motifs with the lowest positional conservation score $P p c<0.25$ was much lower than the number of $R$ motifs. A one sample Wilcoxon signed rank indicated that the $X$ motifs and the $R$ motifs have significantly different medians with two-sided values $p=0.031$ for the mammals and $p=0.016$ for the yeasts.

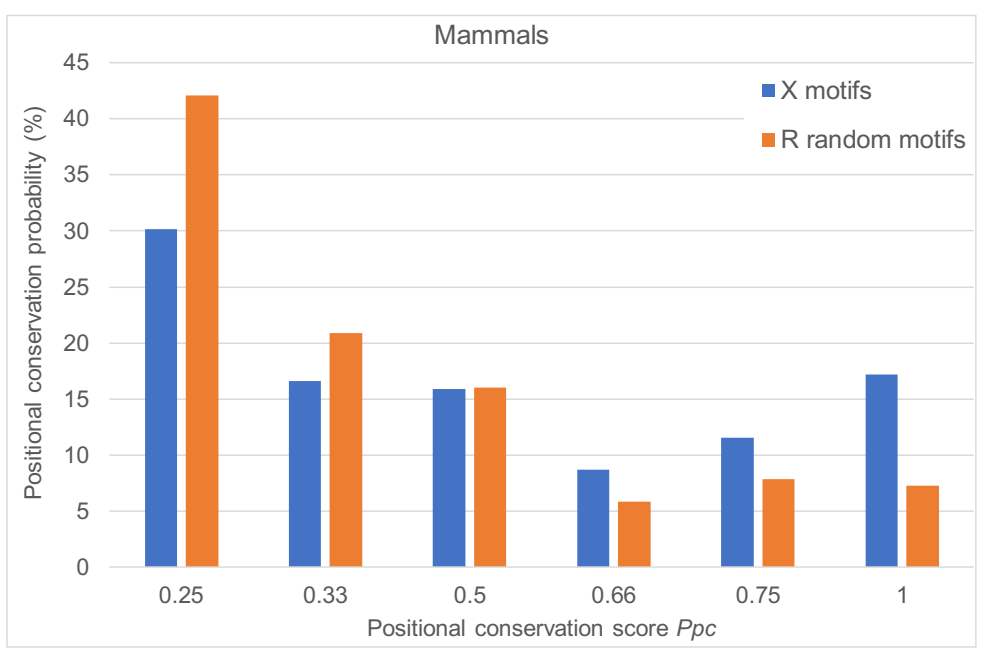

Figure 5. Positional conservation probability (\%) of $X$ motifs and $R$ random motifs in the mammal gene multiple alignments with respect to the human reference genes $\mathbb{H}$ as a function of the score $P p c(m)$ (Definition $10 ; m=(m(\mathcal{S}, \mathbb{H})), \mathcal{S} \in\{X, R\}$ ) varying from 0 (no conservation in the alignment) to 1 (highest conservation in the alignment).

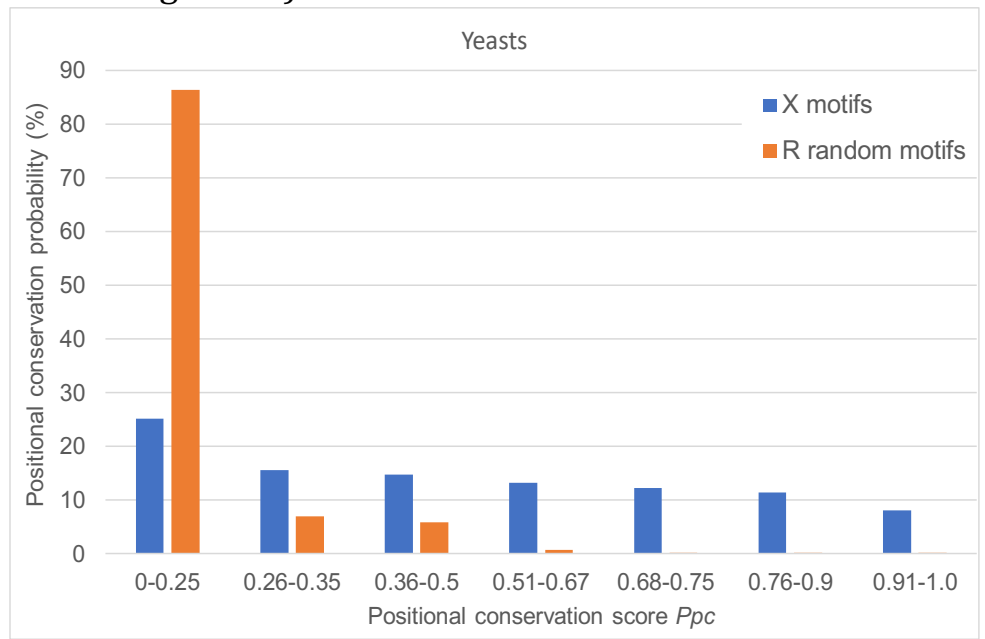

Figure 6. Positional conservation probability (\%) of $X$ motifs and $R$ random motifs in the yeast gene multiple alignments with respect to the $S$. cerevisiae reference genes $\mathbb{C}$ as a function of the score $P p c(m)$ (Definition 10; $m=(m(\mathcal{S}, \mathbb{C})), \mathcal{S} \in\{X, R\})$ varying from 0 (no conservation in the alignment) to 1 (highest conservation in the alignment). 
We conclude that $X$ motifs are more likely to be preserved in the same position in the orthologous genes of mammals and yeasts than $R$ random motifs.

\subsubsection{Sequence conservation of $X$ motifs in mammal and yeast genes}

In the previous section, we showed that $X$ motifs tend to occur at the same positions in orthologous genes from different organisms. To investigate the level of sequence conservation within the $X$ motifs that are found at the same position, we computed several classical pairwise alignment parameters that were defined in Section 2.5 .

We first calculated the percentage $\operatorname{Pid}(m)$ (Definition $11 ; m=(m(\mathcal{S}, \mathbb{R})), \mathcal{S} \in\{X, \bar{X}\}$ ), of identical nucleotides in the aligned $X$ motifs and compared it to the percentage of identical nucleotides in the aligned non- $X$ motifs (alignment columns with no $X$ motifs). For this initial analysis, we selected two organisms from each of the mammal and yeast gene sets. For the mammals, 14,681 gene pairwise alignments containing both human $\mathbb{H}$ and mouse $\mathbb{M}$ genes were used, and for the yeasts, 1088 gene pairwise alignments containing both $S$. cerevisiae $\mathbb{C}$ and $K$. lactis $\mathbb{L}$ genes were used. The Pid observed in $X$ motifs was $87.44 \%$ for $\mathbb{H}-\mathbb{M}$ alignments, and $59.88 \%$ for $\mathbb{C}$ - $\mathbb{L}$ alignments. In comparison, the Pid observed in non- $X$ motifs was $\mathbf{7 7 . 5 6 \%}$ for $\mathbb{H}-\mathbb{M}$ alignments, and $53.94 \%$ for $\mathbb{C}$ - $\mathbb{L}$ alignments. For $\mathbb{H}-\mathbb{M}$ alignments $(n=14,681)$, a $X^{2}$ test shows a strongly significant difference between the Pid values of $X$ motifs (87.44\%) and non- $X$ motifs $(77.56 \%)$ with one-sided value $p \approx 10^{-110}$. For $\mathbb{C}-\mathbb{L}$ alignments $(n=$ 1088), a $X^{2}$ test shows a significant difference between the $P i d$ values of $X$ motifs (59.88\%) and non- $X$ motifs (53.94\%) with a one-sided value $p \approx 0.005$. Thus, the sequences of $X$ motifs are generally more conserved, i.e. evolve more slowly, than the remainder of the gene alignments.

This increased conservation of $X$ motifs indicates that their sequences are maintained during natural selection, and may reflect differences in the strengths of positive selection or purifying selection. To understand the relative contributions of these different modes of selection better, we calculated the ratio $\operatorname{Pns}(m) / P s(m)$ (Definition $15 ; m=(m(\mathcal{S}, \mathbb{R})), \mathcal{S} \in\{X, \bar{X}\}$ ), of non-synonymous to synonymous substitutions for $\mathbb{H}-\mathbb{M}$ and $\mathbb{C}-\mathbb{L}$ alignments (Table 9 and Table 10). This ratio is commonly used to infer purifying $(P n s / P s<1)$ or positive $(P n s / P s>1)$ selection in genes. It is important to note that a nonsynonymous substitution implies a change in the amino acid in the translated protein, while a synonymous substitution only changes the codon: the original codon is replaced by another codon coding for the same amino acid.

\begin{tabular}{|l|c|c|c|c|c|c|c|}
\hline $\mathbb{H}-\mathbb{M}$ alignment & $N n s$ & $N s$ & Ons & Os & Pns & Ps & Pns $/$ Ps \\
\hline$X$ motifs & $1,611,224$ & 480,358 & 99,670 & 184,643 & 0.06 & 0.38 & 0.16 \\
Non- $X$ motifs & $19,772,931$ & $8,225,136$ & $1,524,889$ & $2,572,797$ & 0.08 & 0.31 & 0.25 \\
\hline
\end{tabular}

Table 9. Comparison of non-synonymous and synonymous substitutions for $X$ motifs and non- $X$ motifs in pairs of aligned genes in human $\mathbb{H}$ and mouse $\mathbb{M}$. $N n s(m), N s(m)$ respectively, are the potential numbers of non-synonymous, synonymous respectively, sites for the motifs $m=(m(\mathcal{S}, \mathbb{H})), \mathcal{S} \in\{X, \bar{X}\}$, (Definition 13). Ons $(m), O s(m)$ respectively, are the observed numbers of non-synonymous, synonymous respectively, substitutions of the motifs $m$ (Definition 14). Pns $(m), P s(m)$ respectively, are 
the percentages of non-synonymous, synonymous respectively, substitutions of the motifs $m$ (Definition 15).

\begin{tabular}{|l|c|c|c|c|c|c|c|}
\hline $\mathbb{C}$ - $\mathbb{L}$ alignment & $N n s$ & $N s$ & Ons & Os & Pns & Ps & Pns $/ P s$ \\
\hline$X$ motifs & 369,426 & 93,981 & 103,766 & 80,081 & 0.28 & 0.85 & 0.33 \\
Non- $X$ motifs & $5,310,908$ & $1,973,266$ & $1,580,781$ & $1,362,399$ & 0.30 & 0.69 & 0.43 \\
\hline
\end{tabular}

Table 10. Comparison of non-synonymous and synonymous substitutions for $X$ motifs and non- $X$ motifs in pairs of aligned genes in $S$. cerevisiae $\mathbb{C}$ and $K$. lactis $\mathbb{L}$. $N n s(m), N s(m)$ respectively, are the potential numbers of non-synonymous, synonymous respectively, sites for the motifs $m=(m(\mathcal{S}, \mathbb{C})), \mathcal{S} \in\{X, \bar{X}\}$, (Definition 13). Ons $(m)$, Os $(m)$ respectively, are the observed numbers of non-synonymous, synonymous respectively, substitutions of the motifs $m$ (Definition 14).Pns $(m), P s(m)$ respectively, are the percentages of non-synonymous, synonymous respectively, substitutions of the motifs $m$ (Definition 15).

When we compare the rates of non-synonymous and synonymous substitutions, Pns and Ps respectively, for $\mathbb{H}-\mathbb{M}$ than $\mathbb{C}$ - $\mathbb{L}$, the values of $P n s$ and $P s$ are obviously lower in both $X$ motifs and non$X$ motifs, due to the smaller phylogenetic distance between $\mathbb{H}$ and $\mathbb{M}$. In other words, $\mathbb{H}$ and $\mathbb{M}$ are more closely related than $\mathbb{C}$ and $\mathbb{L}$, so we would expect less substitutions, both synonymous and nonsynonymous. Also, the values of $P n s$ are lower than $P s$ for $X$ motifs and non- $X$ motifs in both sets of genes. Again, this is expected since non-synonymous substitutions have a larger effect on the translated protein and occur less often than synonymous substitutions.

Importantly, we observe significantly lower ratios $P n s / P s$ of non-synonymous to synonymous substitutions in $X$ motifs than in non- $X$ motifs, suggesting more evolutionary constraints on $X$ motifs. Furthermore, while the proportion $P n s$ of non-synonymous substitutions is lower between $X$ motifs and non- $X$ motifs, the proportion $P S$ of synonymous substitutions is higher in $X$ motifs than in non- $X$ motifs. This result motivated the studies presented in the next section, which were designed to analyse in more detail the specific selective constraints in $X$ motifs.

\subsubsection{Synonymous substitutions of trinucleotides in $X$ motifs}

Given a multiple alignment of orthologous gene sequences, our goal is to determine whether trinucleotides in $X$ motifs are conserved beyond what would be expected by chance if they were evolving only under the selective pressure on the amino acid they encode. Therefore, we chose to consider only those positions in the alignment with a conserved amino acid, i.e. involving only synonymous substitutions.

We first calculated the codon substitution matrices $\mathbf{A}(m)$ (defined in Section 2.6) for the $X$ motifs in all the mammal and yeast gene multiple alignments. These two matrices $\mathbf{A}(m)$ are shown in Appendix (Table 19 and Table 20). We then normalized the columns of $\mathbf{A}(m)$ to produce the two normalized codon substitution matrices $\mathbf{B}(m)$ (defined in Section 2.6) for the $X$ motifs, and extracted the rows and columns of the matrices $\mathbf{B}(m)$ that correspond to the synonymous substitutions of the $X$ codons, as shown in Appendix (Table 21 and Table 22). We also calculated the equivalent submatrices $\mathbf{B}(m)$ for the $R$ random motifs. Finally, we calculated the percentages $\operatorname{Paac}(m(X, \mathbb{R}), p)$ (Definition 16) of conservation 
of $X$ codons per amino acid $p \in \mathcal{X}$ in Table 21 and Table 22 , for the two mammal and yeast gene multiple alignments, as summarized in Table 11 and Table 12 . In addition, we provide in these Table 11 and Table $\underline{12}$, the mean percentages $\bar{P} \operatorname{aac}(m(X, \mathbb{R}), \mathcal{X})$ (Definition 17) of conservation of the 12 amino acids $X(2)$ for mammals and yeasts. For comparison, the values of $\operatorname{Paac}(\bar{m}(R, \mathbb{R}), p)$ (Definition 18) and $\bar{P}$ aac $(\bar{m}(R, \mathbb{R}), \mathcal{X})$ (Definition 19) calculated for the $R$ mean random motifs from the 100 random codes $R$ are reported. As these Paac and $\bar{P} a a c$ values for $R$ motifs are mean values, their distributions with a \pm 0.99 confidence interval are shown in Figure 7 and Figure 8.

\begin{tabular}{|c|ccccccccccccc|}
\cline { 2 - 10 } \multicolumn{1}{c|}{} & Mean & $A$ & $D$ & $E$ & $F$ & $G$ & $I$ & $L$ & $N$ & $Q$ & $T$ & $V$ & $Y$ \\
\hline$m(X, \mathbb{H})$ & 78.1 & 66.1 & 89.4 & 90.3 & 78.9 & 77.3 & 84.9 & 78.3 & 85.6 & 80.7 & 63.5 & 65.7 & 76.1 \\
$\bar{m}(R, \mathbb{H})$ & 67.6 & 60.2 & 73.0 & 76.8 & 77.7 & 68.1 & 68.0 & 67.1 & 70.5 & 71.3 & 58.1 & 62.6 & 74.6 \\
\hline
\end{tabular}

Table 11. For the mammal gene multiple alignments with respect to the human reference genes $s_{1}=\mathbb{H}$, mean percentage $\bar{P}$ aac $(m(X, \mathbb{H}), X)$ (Definition 17) of conservation of $X$ codons for the 12 amino acids $X$ (2) coded by the $X$ motifs and percentages $\operatorname{Paac}(m(X, \mathbb{H}), p)$ (Definition 16) of conservation per amino acid $p \in \mathcal{X}$ (first row). Mean percentage $\bar{P} a a c(\bar{m}(R, \mathbb{H}), \mathcal{X})$ (Definition 19) of conservation of $X$ codons for the 12 amino acids $\mathcal{X}(2)$ coded by the $R$ mean random motifs (from the 100 random codes $R$ ) and percentages $\operatorname{Paac}(\bar{m}(R, \mathbb{H}), p$ ) (Definition 18) of conservation per amino acid $p \in \mathcal{X}$ (second row).

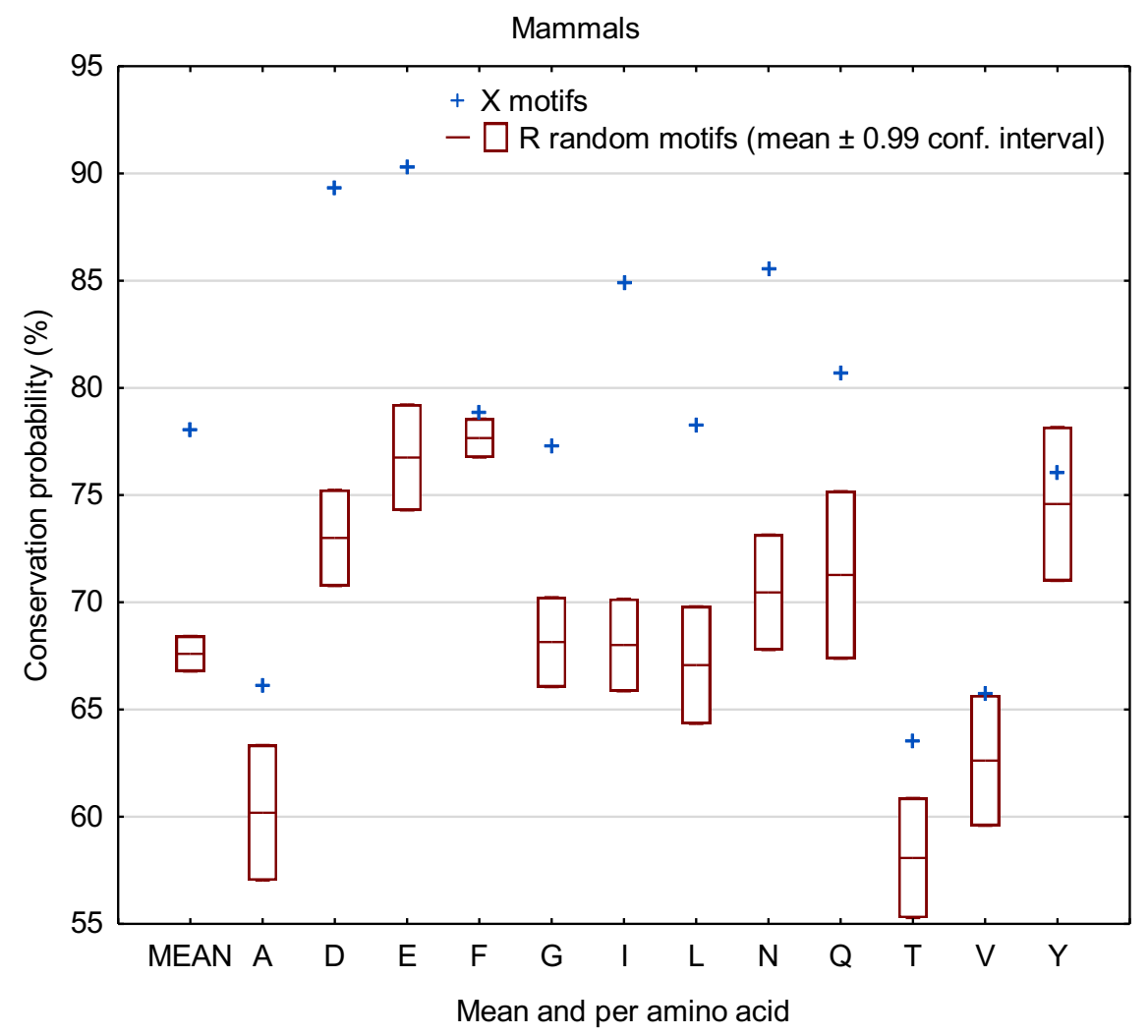

Figure 7 (associated with Table 11). For the mammal gene multiple alignments with respect to the human reference genes $s_{1}=\mathbb{H}$, mean percentage $\bar{P} a a c(m(X, \mathbb{H}), \mathcal{X})$ (Definition 17) of conservation of $X$ codons for the 12 amino acids $X$ (2) coded the $X$ motifs and percentages $\operatorname{Paac}(m(X, \mathbb{H}), p)$ (Definition 16) of conservation per amino acid $p \in \mathcal{X}$ (blue cross). The distribution of the $R$ mean random motifs (from the 100 random codes $R$ ) is indicated by boxplots representing the mean percentage $\bar{P} \operatorname{aac}(\bar{m}(R, \mathbb{H}), \mathcal{X})$ (Definition 19) and percentages $\operatorname{Paac}(\bar{m}(R, \mathbb{H}), p$ ) (Definition 18) with a \pm 0.99 confidence interval. 
A new and strong property is identified with the $X$ motifs of the circular code $X$. The average percentage $(\bar{P} a a c)$ conservation of $X$ codons is significantly higher in $X$ motifs than the conservation observed in the $R$ mean random motifs in the mammal gene alignments (one-sided Student's $t$-test with $p \approx 10^{-55}$ ) (Table 11 and Figure 7). Furthermore, this is true for 11 out of 12 amino acids (percentage Paac). For the amino acid $Y$, the conservation of $X$ codons in $X$ motifs is higher than in $R$ motifs although the difference is not significant at 0.99 . This new property can be formalized simply by the following inequalities:

$$
\left\{\begin{array}{c}
\bar{P} \operatorname{Pac}(m(X, \mathbb{H}), X)>\bar{P} \operatorname{Paac}(\bar{m}(R, \mathbb{H}), X) \\
\operatorname{Paac}(m(X, \mathbb{H}), p)>\operatorname{Paac}(\bar{m}(R, \mathbb{H}), p) \quad \forall p \in X
\end{array}\right.
$$

\begin{tabular}{|l|ccccccccccccc|}
\cline { 2 - 11 } \multicolumn{1}{c|}{} & Mean & $A$ & $D$ & $E$ & $F$ & $G$ & $I$ & $L$ & $N$ & $Q$ & $T$ & $V$ & $Y$ \\
\hline$m(X, \mathbb{C})$ & 29.3 & 16.1 & 45.3 & 41.8 & 29.9 & 40.4 & 39.3 & 10.6 & 33.5 & 13.6 & 14.9 & 33.6 & 33.0 \\
$\bar{m}(R, \mathbb{C})$ & 22.3 & 19.1 & 26.9 & 25.2 & 29.8 & 27.1 & 20.3 & 21.8 & 22.3 & 20.9 & 15.6 & 18.2 & 33.0 \\
\hline
\end{tabular}

Table 12. For the yeast gene multiple alignments with respect to the $S$. cerevisiae reference genes $s_{1}=$ $\mathbb{C}$, mean percentage $\bar{P} a a c(m(X, \mathbb{C}), \mathcal{X})$ (Definition 17) of conservation of $X$ codons for the 12 amino acids $X$ (2) coded by the $X$ motifs and percentages $\operatorname{Paac}(m(X, \mathbb{C}), p)$ (Definition 16) of conservation per amino acid $p \in \mathcal{X}$ (first row). Mean percentage $\bar{P} a a c(\bar{m}(R, \mathbb{C}), \mathcal{X})$ (Definition 19) of conservation of $X$ codons for the 12 amino acids $\mathcal{X}(2)$ coded by the $R$ mean random motifs (from the 100 random codes $R$ ) and percentages $\operatorname{Paac}(\bar{m}(R, \mathbb{C}), p$ ) (Definition 18) of conservation per amino acid $p \in \mathcal{X}$ (second row).

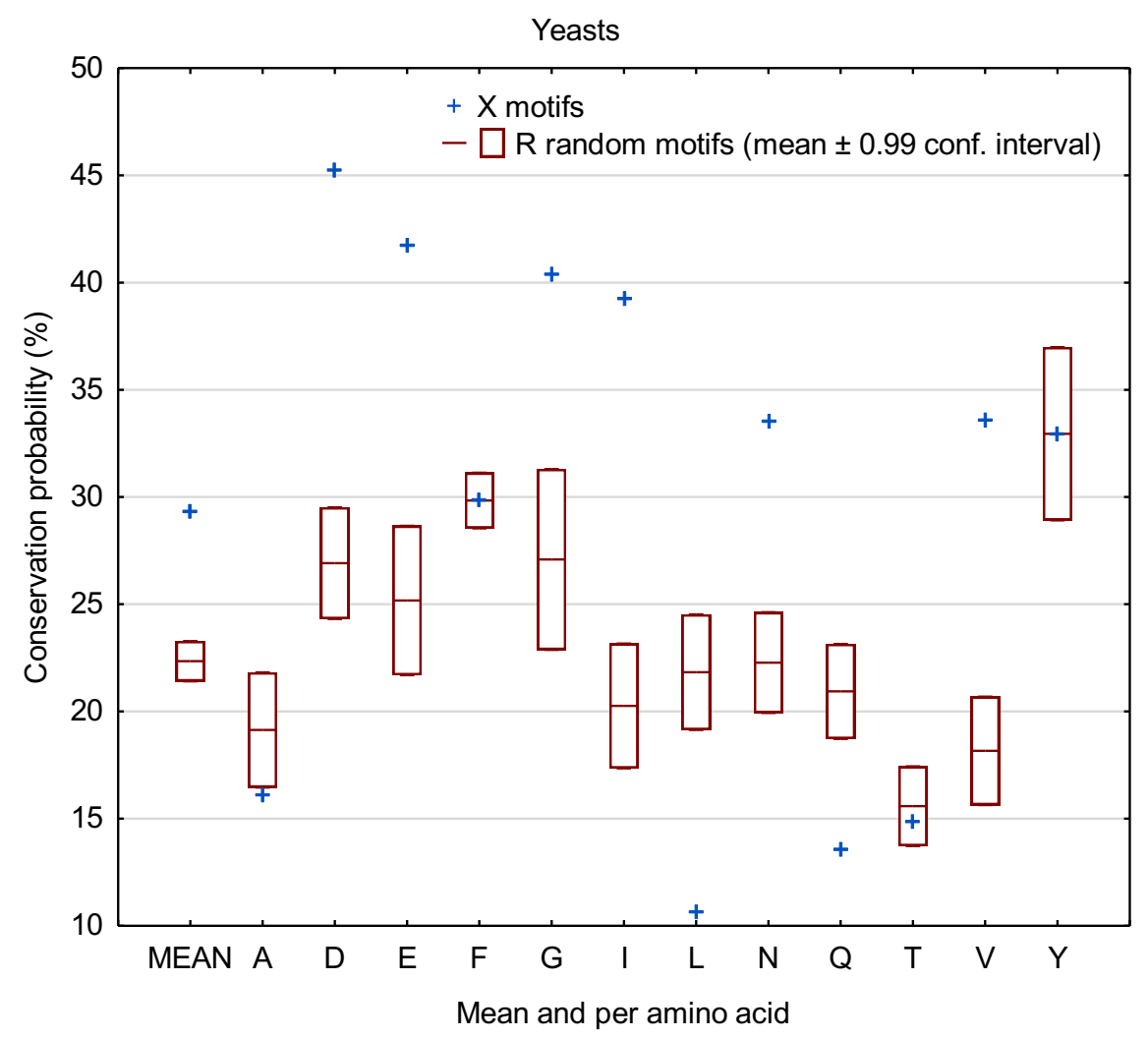

Figure 8 (associated with Table 12). For the yeast gene multiple alignments with respect to the $S$. cerevisiae reference genes $s_{1}=\mathbb{C}$, mean percentage $\bar{P} a a c(m(X, \mathbb{C}), \mathcal{X})$ (Definition 17) of conservation of $X$ codons for the 12 amino acids $X$ (2) coded by the $X$ motifs and percentages $\operatorname{Paac}(m(X, \mathbb{C}), p)$ (Definition 16) of conservation per amino acid $p \in \mathcal{X}$ (blue cross). The distribution of the $R$ mean random motifs (from the 100 random codes $R$ ) is indicated by boxplots representing the mean 
percentage $\bar{P} a a c(\bar{m}(R, \mathbb{C}), \mathcal{X})$ (Definition 19) and percentages $\operatorname{Paac}(\bar{m}(R, \mathbb{C}), p)$ (Definition 18) with a \pm 0.99 confidence interval.

The average percentage $(\bar{P} a a c)$ conservation of $X$ codons is significantly higher in $X$ motifs than in the $R$ mean random motifs in the yeast gene alignments (one-sided Student's $t$-test with $p \approx 10^{-35}$ ) (Table $\underline{12}$ and Figure 8). For 6 out of 12 amino acids, the conservation (percentage Paac) of $X$ codons in $X$ motifs is higher than in $R$ motifs. In contrast, for the amino acids $A, L, Q$ and $T$, the conservation is lower than in the $R$ motifs. For the amino acids $F$ and $L$, the conservation is similar to the $R$ motifs. This property can also be summarized by the following inequalities:

$$
\left\{\begin{array}{c}
\bar{P} \operatorname{aac}(m(X, \mathbb{C}), X)>\bar{P} \operatorname{aac}(\bar{m}(R, \mathbb{C}), X) \\
\operatorname{Paac}(m(X, \mathbb{C}), p)>\operatorname{Paac}(\bar{m}(R, \mathbb{C}), p) \quad \forall p \in X \backslash\{A, L, Q, T\}
\end{array}\right.
$$

The conservation values of the motifs observed in the yeast alignments is lower than in the human alignments. This is not surprising since it is known that the yeasts diverged much earlier (more synonymous and non-synonymous substitutions) than the mammals included in this study. This evolutionary diversity in yeasts may also explain the exception with the four amino acids observed with this simple statistical parameter Paac. It should also be stressed that the identified conservation property of $X$ codons in $X$ motifs with respect to the amino acids is independent of the codon usage, the $G C$ content, the nucleotide composition, the length of genes, etc.

\subsubsection{A new hypothesis of evolution of the genetic code: union of circular codes associated with each amino acid}

The statistical analyses performed in the previous sections show that the 20 trinucleotides of the circular code $X$ (1) are strongly linked to the amino acids they encode, thus, leading to a partition of the 20 trinucleotides of $X$ into 12 trinucleotide classes, each trinucleotide class being associated with an amino acid $p \in \mathcal{X}(2)$. This property leads us to propose that the extant genetic code may result from a union of circular codes: the subcodes of the circular code $X(1)$ associated with each amino acid (Table $\underline{13}$ and Figure 9). Remember that a subcode of a circular code, is also circular. Interestingly, classes of circular codes with the strongest constraints of reading frame retrieval, i.e. the strong comma-free and comma-free codes (Theorem 3 and Theorem 2), can code an amino acid. Using these theorems, we determine the circular class of each trinucleotide code involved in Table 13 (an initial approach developed in Michel, 2014, Section 3.4, Table 6).

The evolution of the genetic code may thus have started from the circular codes with the strongest constraints, i.e. the strong comma-free codes and the comma-free codes with motifs retrieving the reading frame after the reading of 2 and 3 nucleotides, i.e. a nucleotide length of a codon or anticodon. It is tempting to suggest that these circular codes may have emerged independently in different "primitive soups". However, such strongly constrained coding systems may not have been viable in the long term. By relaxing the constraints, they may have evolved to circular codes having flexible motifs for retrieving the reading frame after the reading of at most 13 nucleotides, and to non-circular codes 
without the ability to retrieve the reading frame. Among the 12 amino acids $\mathcal{X}(2)$ coded by the circular code $X$ (1), 10 amino acids are coded by strong comma-free codes and 2 amino acids $E_{X}$ and $L_{X}$ of $X$, by comma-free codes (Table 13 and Figure 9 ). In the extant genetic code, only 3 amino acids $D, N$ and $Q$ are still coded by strong comma-free codes, 6 amino acids $A, E, I, T, V$ and $Y$, by comma-free codes, 1 amino acid $L$, by a circular code, and 2 amino acids $F$ and $G$, by simple codes (not circular). The union of circular codes allows to extend the amino acid coding. For example, the union of the strong comma-free code $Q_{X}=\{C A G\}$ of $X$ and the strong comma-free code $\{C A A\}$ leads to the strong comma-free code $Q=$ $\{C A A, C A G\}$ of the genetic code, etc. Obviously, the union of 2 comma-free codes does not imply that the resulting code is comma-free, see for example the case of the amino acid $L$ (Table 13). The 8 remaining amino acids could have been generated by mutations in circular codes. The extant genetic code is a code from a mathematical point of view (Definition 3), however it is not circular, i.e. it does not have the ability to retrieve the reading frame in genes after its circularity property loss.

\begin{tabular}{|c|c|c|c|c|c|}
\hline AA $\quad$ Circular code $X$ & Class & Union & Class & Genetic code & Class \\
\hline Asn $N_{X}=\{A A C, A A T\}$ & $S C F$ & & & $N=\{A A C, A A T\}$ & $S C F$ \\
\hline Asp $D_{X}=\{G A C, G A T\}$ & $S C F$ & & & $D=\{G A C, G A T\}$ & $S C F$ \\
\hline $\operatorname{Gln} Q_{X}=\{C A G\}$ & $S C F$ & $\{C A A\}$ & $S C F$ & $Q=\{C A A, C A G\}$ & $S C F$ \\
\hline Glu $E_{X}=\{G A A, G A G\}$ & $C F$ & & & $E=\{G A A, G A G\}$ & $C F$ \\
\hline Phe $F_{X}=\{T T C\}$ & $S C F$ & $\{T T T\}$ & NC & $F=\{T T C, T T T\}$ & NC \\
\hline Tyr $Y_{X}=\{T A C\}$ & $S C F$ & $\{T A T\}$ & $C F$ & $Y=\{T A C, T A T\}$ & $C F$ \\
\hline Ile $\quad I_{X}=\{A T C, A T T\}$ & $S C F$ & $\{A T A\}$ & $C F$ & $I=\{A T A, A T C, A T T\}$ & $C F$ \\
\hline Ala $A_{X}=\{G C C\}$ & $S C F$ & $\{G C A, G C G, G C T\}$ & $C F$ & $A=\{G C A, G C C, G C G, G C T\}$ & $C F$ \\
\hline Gly $G_{X}=\{G G C, G G T\}$ & $S C F$ & $\{G G A, C$ & $N C$ & $G=\{G G A, G G C, G G G, G G T\}$ & NC \\
\hline Thr $T_{X}=\{A C C\}$ & $S C F$ & $\{A C A, A C G, A C T\}$ & $C F$ & $T=\{A C A, A C C, A C G, A C T\}$ & $C F$ \\
\hline Val $V_{X}=\{G T A, G T C, G T T\}$ & $S C F$ & $\{G T G\}$ & $C F$ & $V=\{G T A, G T C, G T G, G T T\}$ & $C F$ \\
\hline Leu $L_{X}=\{C T C, C T G\}$ & $C F$ & $\{C T A, C T T, T T A, T T G\}$ & $C F$ & $L=\{C T A, C T C, C T G, C T T, T T A, T T G\}$ & $C$ \\
\hline
\end{tabular}

Table 13. Classes of codes (non-circular $N C$, circular $C$, comma-free $C F$, strong comma-free $S C F$ ) of the 12 amino acids $\mathcal{X}(2)$ with respect to the circular code $X$ (1) and the universal genetic code.

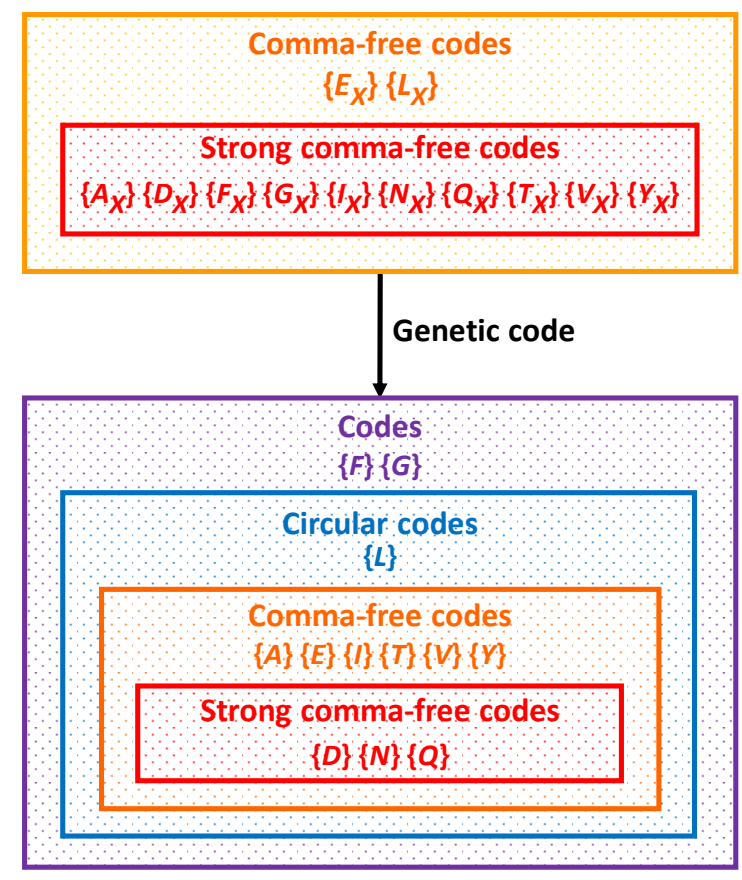

Figure 9 (associated with Table 13). Evolution of the genetic code by union of circular codes associated with each amino acid from the circular code $X$ (1). 


\subsection{Functionality of $X$ motifs in extant genomes}

In the previous section, we identified specific evolutionary constraints, suggesting that the $X$ motifs in the genomes included in this study have evolved under purifying selection. Indeed, the nucleotides in the $X$ motifs display a considerable excess of synonymous substitutions compared to the non- $X$ motifs. Furthermore, the average conservation of codons in $X$ motifs is significantly higher than expected if the substitution process was random. These results suggest a possible functional role of $X$ motifs, presumably as elements of the complex genome decoding system. In order to investigate the potential effects of $X$ motifs on the translation of protein-coding genes, we compared the frequency of $X$ motifs in the genes of the four mammalian and nine yeast species with existing experimental data on protein expression and protein production. We will show that the experimental data can generally be explained by circular code motifs, i.e. motifs having the property of reading frame retrieval.

\subsubsection{Dicodons associated with reduced protein production are not located in $X$ motifs}

Recently, experimental studies in S. cerevisiae (Gamble et al., 2016) were performed to investigate the effects of different codons on translation efficiency. The authors measured the expression levels of more than 35,000 synthetic protein variants in which three adjacent codons of the coding sequence were randomized. No individual codons had consistent effects on gene expression. However, 17 pairs of adjacent codons (called in the following dicodons) were identified that, when they were present inframe in the coding sequence, reduced the expression level of the genes. This list is recalled in Table 14. In this list, we identified the codons belonging to the circular code $X$.

\begin{tabular}{|llll|}
\hline Dicodon & Class & Dicodon & Class \\
\hline AGGCGA & $N N$ & CGAGCG & $N N$ \\
AGGCGG & $N N$ & CTCCCG & $X N$ \\
ATACGA & $N N$ & CTGATA & $X N$ \\
ATACGG & $N N$ & CTGCCG & $X N$ \\
CGAATA & $N N$ & CTGCGA & $X N$ \\
CGACCG & $N N$ & GTACCG & $X N$ \\
CGACGA & $N N$ & GTACGA & $X N$ \\
CGACGG & $N N$ & GTGCGA & $N N$ \\
CGACTG & $N X$ & & \\
\end{tabular}

Table 14. List of the 17 dicodons that reduced the expression level of the genes (Gamble et al., 2016) (1st and 3rd columns). Class of the dicodons according to its codons belonging to the circular code $X$ (symbol $X$ ) or not (symbol $N$ ) (2nd and 4th columns).

Surprisingly, none of these 17 dicodons are composed of two $X$ codons meaning that they cannot be located in a $X$ motif.

\subsubsection{Correlation of $X$ motifs with dicodons associated with low and high protein production}

Following the work of Gamble et al. (2016), Diambre (2017) performed a statistical analysis of dicodon usage frequencies over two sets of proteins: a low protein abundance (PA) set and a high PA set, from 
nine diverse organisms including three prokaryotes, one plant, one yeast ( $S$. cerevisiae), and two multicellular eukaryotes and two mammals. The working hypothesis was that sequences encoding abundant proteins should be optimized, in the sense of translation efficiency. He found an important bias of dicodon usage depending on PA and determined which dicodons where statistically associated with low or high abundance. These usage preferences cannot be explained by the frequency usage of the single codons. The statistical analysis of coding sequences of nine organisms reveals that in many cases dicodon preferences are shared between related organisms.

\begin{tabular}{|llll|}
\hline Dicodon & Class & Dicodon & Class \\
\hline AAAATA & $N N$ & CAGAAA & $X N$ \\
AATGCA & $X N$ & GAAAGT & $X N$ \\
AATTGG & $X N$ & GAACTA & $X N$ \\
AGTAAG & $N N$ & GCATTT & $N N$ \\
AGTGTG & $N N$ & TATAAA & $N N$ \\
ATAGGT & $N X$ & TATCCG & $N N$ \\
ATTAAA & $X N$ & TTTCAG & $N X$ \\
CAAAGT & $N N$ & TTTTTT & $N N$ \\
\hline
\end{tabular}

Table 15. List of the 16 dicodons with low protein abundance (Diambra, 2017) (1st and 3rd columns). Class of the dicodons according to its codons belong to the circular code $X$ (symbol $X$ ) or not (symbol $N$ ) (2nd and 4th columns).

In addition to the 17 previous dicodons (identified in S. cerevisiae), this study identified 16 new dicodons (Table 15) associated with low protein abundance in a number of different organisms. Again, these 16 dicodons cannot be located in a $X$ motif. Thus, there are 33 low abundance dicodons that support the circular code theory.

Furthermore, the study revealed 40 dicodons shared between different organisms and preferentially used by high abundance proteins (Table 16). Importantly, 27 of these 40 dicodons (67.5\%) are potentially in $X$ motifs.

\begin{tabular}{|c|c|c|c|c|c|c|c|}
\hline Dicodon & Class & Dicodon & Class & Dicodon & Clas & Dicodc & Clas \\
\hline$A A C A A C$ & $X X$ & ACCTTC & $X X$ & GACACC & $X X$ & GTCACC & $X X$ \\
\hline$A A C A A G$ & $X N$ & ATCAAC & $X X$ & GACTAC & $X X$ & GTCATC & $X X$ \\
\hline AACACC & $X X$ & $A T C A A G$ & $X N$ & GATGCT & $X N$ & GTTGCC & $X X$ \\
\hline AAGTCC & $N N$ & АTCACC & $X X$ & GCCAAC & $X X$ & TACAAC & $X X$ \\
\hline ACCAAC & $X X$ & ATCATC & $X X$ & $G C C A A G$ & $X N$ & $T A C A A G$ & $X N$ \\
\hline$A C C A A G$ & $X N$ & ATTGCC & $X X$ & GCCACC & $X X$ & ТССАCС & $N X$ \\
\hline АCCACC & $X X$ & ССАCСA & $N N$ & GCCATC & $X X$ & TTCAAC & $X X$ \\
\hline ACCATC & $X X$ & CGTCGT & $N N$ & GCCGCC & $X X$ & TTCAAG & $X N$ \\
\hline ACCATT & $X X$ & $G A C A A C$ & $X X$ & GGTGTC & $X X$ & ТTCACC & $X X$ \\
\hline ACCGCC & $X X$ & $G A C A A G$ & $X N$ & GTCAAG & $X N$ & TTCATC & $X X$ \\
\hline
\end{tabular}

Table 16. List of the 40 dicodons with high protein abundance (Diambra, 2017) (1st, 3rd, 5th and 7th columns). Class of the dicodons according to its codons belong to the circular code $X$ (symbol $X$ ) or not (symbol $N$ ) (2nd, 4th, 6th and 8th columns). 


\subsubsection{Classification of genes as low or high abundance according to the circular code theory}

The experimental and statistical results of Gamble et al. (2016) and Diambre (2017) (Table 14, Table 15 and Table 16) can be summarized in the following Table 17.

\begin{tabular}{|c|cc|c|}
\cline { 2 - 4 } \multicolumn{1}{c|}{} & $X X$ & $\{N N, N X, X N\}$ & Total \\
\hline Low abundance protein & 0 & 33 & 33 \\
High abundance protein & 27 & 13 & 40 \\
\hline Total & 27 & 46 & 73 \\
\hline
\end{tabular}

Table 17. Contingency table of low/high abundance protein and presence/absence of dicodons $X X$ (deduced from Table 14, Table 15 and Table 16).

A $X^{2}$ test shows a strongly significant relation between the presence/absence of dicodons $X X$ and protein abundancy with a one sided value $p \approx 10^{-9}$ (Table 17). The following probabilities can be easily deduced from Table 17:

$$
\begin{gathered}
P(\text { Low abundance protein } \mid X X)=0 / 33=0 \%, \\
P(\text { High abundance protein } \mid X X)=27 / 40=67.5 \% .
\end{gathered}
$$

Thus, the presence-absence of $X X$ dicodons in a gene is an important and new factor in the classification of genes as low or high abundance.

\subsubsection{Presence of $X$ motifs in wild type genes and genes optimized to increase expression}

The SGDB database (Wu et al., 2007) contains gene expression data for genes that have been experimentally re-engineered to increase gene expression. Generally, this is achieved by replacing codons in the wild type gene with optimal codons for the expression system (i.e. replace rare codons with the most frequently used codons in the organism). We only considered the re-engineered genes that did not involve non-synonymous changes. Thus, we analysed 42 re-engineered genes that had increased expression and 4 re-engineered genes had no significant increase in expression. We searched for $X$ motifs and $R$ random motifs (from the 100 random codes) in the wild type genes and the genes optimized for gene expression. Then, we calculated the mean number and the mean nucleotide length

\begin{tabular}{|c|c|c|c|c|c|}
\hline & & $\begin{array}{c}\text { Mean number } \\
\text { of } X \text { motifs }\end{array}$ & $\begin{array}{c}\text { Mean number } \\
\text { of } R \text { motifs }\end{array}$ & $\begin{array}{l}\text { Mean length } \\
\text { of } X \text { motifs }\end{array}$ & $\begin{array}{c}\text { Mean length } \\
\text { of } R \text { motifs }\end{array}$ \\
\hline 42 genes with & Wild type & 5.4 & 3.6 & 86.1 & 53.7 \\
\hline increased expression & Optimized gene & 11.2 & 3.7 & 188.6 & 58.2 \\
\hline 3 genes with no & Wild type & 5.3 & 2.6 & 80.0 & 35.8 \\
\hline increased expression & Optimized gene & 5.0 & 3.8 & 80.0 & 55.6 \\
\hline
\end{tabular}
of $X$ and $R$ motifs per sequence (Table 18).

Table 18. Mean number and mean nucleotide length of $X$ and $R$ random motifs per wild type gene and per optimized gene from the SGDB database (Wu et al., 2007).

For the re-engineered genes that did not present an increase expression, we observe a non-significant difference in the mean number $(5.0-5.3=-0.3$, one tailed Wilcoxon test with value $p=0.50)$ and no 
difference in the mean length $(80.0-80.0=0)$ of $X$ motifs between the optimized genes and the wild type genes. These differences are also not significant for $R$ motifs $(3.8-2.6=1.2$ and $55.6-35.8=$ 19.8, respectively, data not shown). In contrast, for the re-engineered genes that resulted in increased expression, the optimized genes have significantly more $X$ motifs $(11.2-5.4=5.8$, one tailed Wilcoxon test with value $\left.p \approx 10^{-6}\right)$ and the $X$ motifs covered a larger proportion of the genes $(188.6-86.1=$ 102.5 , one tailed Wilcoxon test with value $p \approx 10^{-6}$ ) for most genes (Figure 10). These differences are not observed with the $R$ motifs (one tailed Wilcoxon test, $p$ values equal to 0.24 and 0.12 , respectively). Thus, this important result suggest a potential new strategy for the efficient gene optimization.

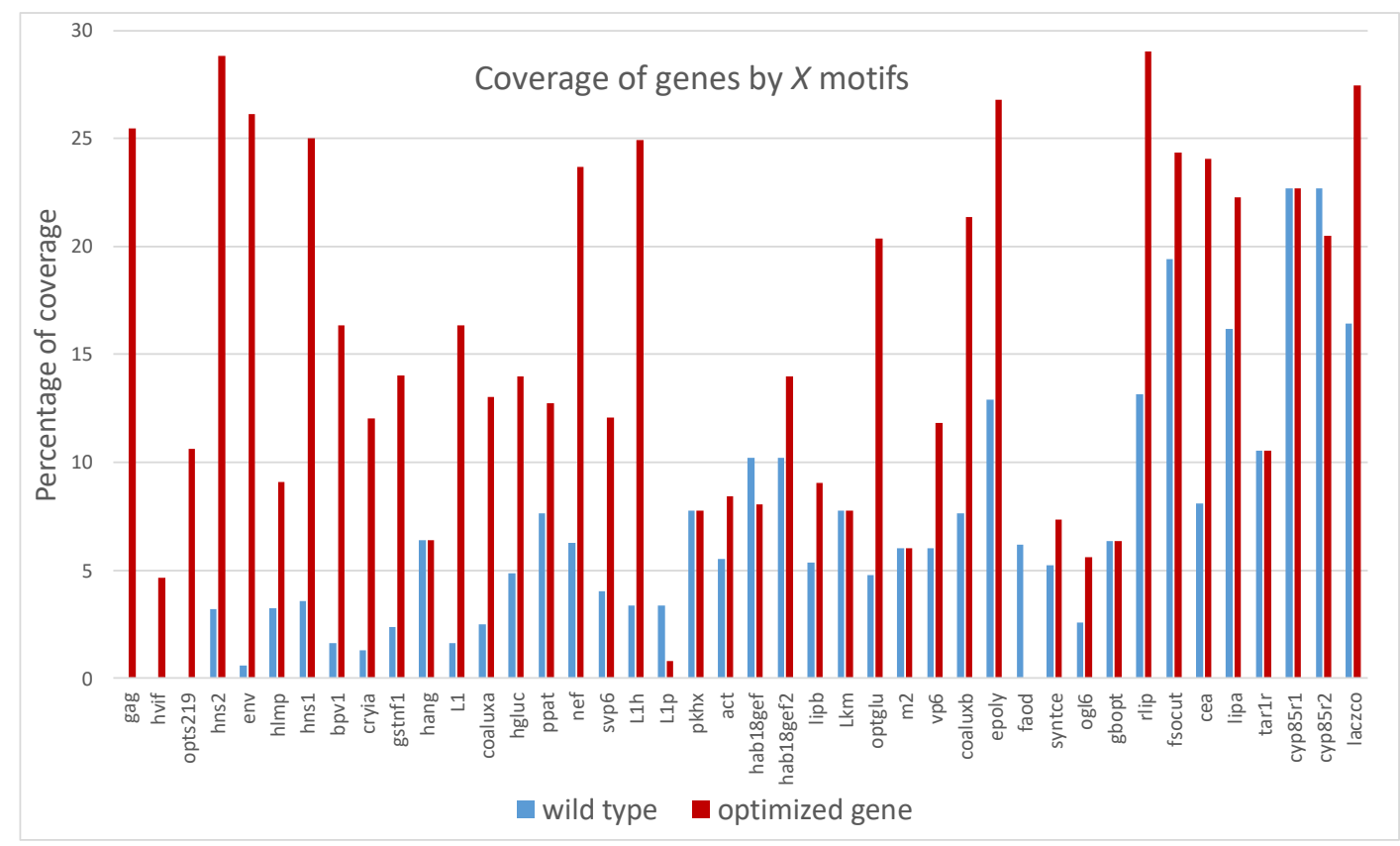

Figure 10. Percentage coverage (total length of $X$ motifs divided by the total length of genes) of 42 wild type and optimized genes by $X$ motifs.

\section{Conclusion}

The work described in this paper addressed two questions: are $X$ motifs conserved during evolution? and do they continue to play a functional role in the processes of genome decoding and protein synthesis?

We performed a large scale study involving the complete genomes of four mammals and nine yeast species. The organisms chosen represent a large phylogenetic distribution, and a wide variety of gene structures, ranging from the simple, single exon genes of $S$. cerevisiae to the highly complex intron/exon structure of human genes. To avoid any bias towards a specific sequence alignment algorithm or evolutionary model, the multiple alignments of the gene sequences were obtained by two different methods. First, high quality mammal gene alignments were obtained from a previous independent study (Sharma and Hiller, 2017) of genome annotation methods. Second, multiple alignments of the yeast genes were constructed using a simple protein alignment method (ClustalW, Thompson et al., 1994). 
Furthermore, well characterized, well annotated genomes (Human and S. cerevisiae) were chosen to ensure high quality gene models.

In a preliminary analysis, we identified a strongly significant enrichment of $X$ motifs (number and length) in both mammal and yeast genes, confirming our previous findings in S. cerevisiae (Michel et al., 2017). We then calculated a number of different measures of evolutionary conservation, and showed that the $X$ motifs are more conserved than the rest of the gene sequences, with a lower ratio Pns $P s$ of non-synonymous to synonymous substitutions, indicative of purifying selection. These results were found to hold in both the mammal and yeast gene alignments. We then performed a more in-depth investigation of the synonymous substitutions in $X$ motifs. At this stage, we modelled the evolutionary processes at the codon level, since it is important to account precisely for the protein-coding constraints on each nucleotide site. We demonstrated that the sequence conservation observed in the $X$ motifs is the result of two types of selective pressure. The first type is the pressure to maintain the amino acids of the proteins encoded by the genes. The second type of selective pressure applies only to $X$ motifs and highlights a new conservation property of $X$ motifs per amino acid, which led us to propose a novel hypothesis for the evolution of the genetic code as a union of circular codes associated with each amino acid.

The increased conservation of $X$ motifs and the specific evolutionary constraints suggest that $X$ motifs may represent an additional, overlapping function within the protein-coding regions of genomes. Indeed, the genetic code establishes the rules to translate the 64 possible codons into the 20 amino acids and a stop signal. It is well known that the genetic code is degenerate and that different synonymous codons encoding the same amino acid are not used with the same frequency in different species. The genetic code also contains information that influences the rate and efficiency of translation, although the mechanisms of codon-mediated regulation are still not clear (Brule and Greyhack, 2017). Many recent studies have been performed to try to explain the different codon usages observed and their effects on translation. In particular, it has been shown that the efficiency of translating a particular codon is influenced by the nature of the immediately adjacent flanking codons (Gamble et al., 2016; Diambra, 2017; Chevance and Hughes, 2017). These studies are mostly based on statistical and/or experimental analyses of gene sequences without being related to the results to a theoretical model. Here, we have investigated the pertinence of the circular code theory to explain the observations.

For example, in two related studies (Gamble et al., 2016; Diambra, 2017), a total of 33 dicodons were found to be associated with low protein abundance, and 40 dicodons associated with high abundance proteins. We identified a significant correlation between the protein abundance level and dicodons belonging to the circular code $X$. To further investigate this link between the presence of $X$ motifs in a gene and the expression level, we compared a set of re-engineered genes (that had been experimentally optimized for increased expression, using synonymous substitutions to replace rare codons with more frequent ones) with the original wild type genes. Again, we identified a significant correlation between the number and length of $X$ motifs and protein expression levels. These results, taken together, suggest 
that increasing the proportion of $X$ motifs in a gene may represent an important new strategy for their efficient optimization.

The molecular mechanisms underlying the functional correlations observed here remain to be elucidated. However, it has been observed previously that short $X$ motifs have also been conserved in many transfer RNAs (tRNAs) and ribosomal RNAs (rRNAs) (Michel, 2012, 2013; El Soufi and Michel, 2014, 2015). In particular, the universally conserved nucleotides A1492, A1493 G530 in the ribosome decoding center are located in short $X$ motifs. Given the self-complementary property of the circular code $X$, it is possible that there is some kind of interaction between the $X$ motifs in the protein-coding genes and the ribosomal $X$ motifs in order to maintain the correct reading frame during translation.

The results presented here indicate that the circular code motifs may explain how the choice of different synonymous codons within genes and between species, known as codon usage bias, impacts nucleic acid stability, protein levels, structure, function and evolution. Further investigation, and in vitro or in vivo experimental validation, will be required to refine our hypothesis for the evolution of the genetic code and the proposed functional role in the translation of genes. 


\section{REFERENCES}

Altschul S.F., Madden T.L., Schäffer A.A., Zhang J., Zhang Z., Miller W., Lipman D.J. (1997). Gapped BLAST and PSI-BLAST: a new generation of protein database search programs. Nucleic Acids Research 125, 3389-3402.

Arquès D.G., Michel C.J. (1996). A complementary circular code in the protein coding genes. Journal of Theoretical Biology 182, 45-58.

Brule C.E., Grayhack E.J. (2017). Synonymous codons: choose wisely for expression. Trends in Genetics 33, 283-297.

Chevance F.F.V., Hughes K.T. (2017). Case for the genetic code as a triplet of triplets. Proceedings of the National Academy of Sciences U.S.A., 1614896114.

Diambra L.A. (2017). Differential bicodon usage in lowly and highly abundant proteins. PeerJ 5:e3081.

El Houmami N., Seligmann H. (2017). Evolution of nucleotide punctuation marks: from structural to linear signals. Frontiers in Genetics 8, 36.

El Soufi K., Michel C.J. (2014). Circular code motifs in the ribosome decoding center. Computational Biology and Chemistry 52, 9-17.

El Soufi K., Michel C.J. (2015). Circular code motifs near the ribosome decoding center. Computational Biology and Chemistry 59, 158-176.

El Soufi K., Michel C.J. (2017). Unitary circular code motifs in genomes of eukaryotes. Biosystems 153, 45-62.

Fimmel E., Michel C.J., Strüngmann L. (2016). $n$-Nucleotide circular codes in graph theory. Philosophical Transactions of the Royal Society A: Mathematical, Physical and Engineering Sciences 374, 20150058.

Fimmel E., Michel C.J., Strüngmann L. (2017). Strong comma-free codes in genetic information. Bulletin of Mathematical Biology 79, 1796-1819.

Fimmel E., Strüngmann L. (2018). Mathematical fundamentals for the noise immunity of the genetic code. Biosystems 164, 186-198.

Gamble C.E., Brule C.E., Dean K.M., Fields S., Grayhack E.J. (2016). Adjacent codons act in concert to modulate translation efficiency in yeast. Cell 166, 679-690.

Michel C.J. (2008). A 2006 review of circular codes in genes. Computer and Mathematics with Applications 55, 984-988.

Michel C.J. (2012). Circular code motifs in transfer and 16S ribosomal RNAs: A possible translation code in genes. Computational Biology and Chemistry 37, 24-37.

Michel C.J. (2013). Circular code motifs in transfer RNAs. Computational Biology and Chemistry 45, 17 29.

Michel C.J. (2014). A genetic scale of reading frame coding. Journal of Theoretical Biology 355, 83-94.

Michel C.J. (2015). The maximal $C^{3}$ self-complementary trinucleotide circular code $X$ in genes of bacteria, eukaryotes, plasmids and viruses. Journal of Theoretical Biology 380, 156-177. 
Michel C.J. (2017). The maximal $C^{3}$ self-complementary trinucleotide circular code $X$ in genes of bacteria, archaea, eukaryotes, plasmids and viruses. Life 7, 20, 1-16.

Michel C.J., Nguefack Ngoune V., Poch O., Ripp R., Thompson J.D. (2017). Enrichment of circular code motifs in the genes of the yeast Saccharomyces cerevisiae. Life 7, 52, 1-20.

Nei M., Gojobori T. (1986). Simple methods for estimating the numbers of synonymous and nonsynonymous nucleotide substitutions. Molecular Biology Evolution 3, 418-26.

Seligmann H., Pollock DD. (2004). The ambush hypothesis: hidden stop codons prevent off-frame gene reading. DNA and Cell Biology 23, 701-705.

Seligmann H. (2011). Error compensation of tRNA misacylation by codon-anticodon mismatch prevents translational amino acid misinsertion. Computational Biology and Chemistry 35, 81-95.

Seligmann H., Warthi G. (2017). Genetic Code Optimization for Cotranslational Protein Folding: Codon Directional Asymmetry Correlates with Antiparallel Betasheets, tRNA Synthetase Classes. Computational and Structural Biotechnology Journal 15, 412-424.

Sharma V., Hiller M. (2017). Increased alignment sensitivity improves the usage of genome alignments for comparative gene annotation. Nucleic Acids Research 45, 8369-8377.

Thompson J.D., Higgins D.G., Gibson T.J. (1994). CLUSTAL W: improving the sensitivity of progressive multiple sequence alignment through sequence weighting, position-specific gap penalties and weight matrix choice. Nucleic Acids Research.22, 4673-4680.

Wu G., Zheng Y., Qureshi I., Zin H.T., Beck T., Bulka B., Freeland S.J. (2007). SGDB: a database of synthetic genes re-designed for optimizing protein over-expression. Nucleic Acids Research 35, D76-D79. 


\begin{tabular}{|c|c|c|c|c|c|c|c|c|c|c|c|c|c|c|c|c|c|c|c|c|}
\hline & $N$ & $N$ & $T$ & $I$ & $I$ & $Q$ & $L$ & $L$ & $E$ & $\bar{D}$ & $E$ & $\bar{D}$ & $A$ & $G$ & $G$ & $V$ & 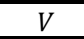 & $V$ & $Y$ & $F$ \\
\hline & $A A C$ & $A A T$ & $\overline{A C C}$ & $A T C$ & ATT & $C A G$ & CTC & CTG & $G A A$ & $G A C$ & $G A G$ & GAT & $G C C$ & $\overline{G G C}$ & GGT & GTA & $G T C$ & GTT & $T A C$ & TTC \\
\hline \begin{tabular}{|l|l|}
$K$ & $A A A$ \\
\end{tabular} & 521 & 569 & 81 & 33 & 22 & 378 & 11 & 23 & 1449 & 126 & 494 & 125 & 21 & 39 & 21 & 35 & 10 & 7 & 14 & 3 \\
\hline$N \mid A A C$ & 74934 & 20002 & 767 & 186 & 53 & 92 & 36 & 10 & 105 & 1728 & 200 & 569 & 199 & 471 & 129 & 3 & 47 & 16 & 178 & 19 \\
\hline$K A A G$ & 650 & 744 & 109 & 31 & 37 & 2148 & 15 & 96 & 423 & 130 & 2316 & 133 & 45 & 40 & 42 & 23 & 9 & 9 & 14 & 3 \\
\hline$N \mid A A T$ & 16365 & 56175 & 200 & 40 & 194 & 131 & 8 & 7 & 147 & 477 & 221 & 1539 & 58 & 112 & 275 & 5 & 8 & 46 & 53 & 5 \\
\hline$T A C A$ & 110 & 103 & 5453 & 143 & 139 & 61 & 11 & 124 & 147 & 16 & 112 & 15 & 387 & 20 & 14 & 264 & 63 & 56 & 3 & 17 \\
\hline$T A C C$ & 905 & 268 & 67464 & 1434 & 369 & 22 & 126 & 50 & 26 & 149 & 45 & 37 & 3797 & 184 & 44 & 27 & 516 & 103 & 24 & 79 \\
\hline$T A C G$ & 57 & 64 & 5203 & 126 & 99 & 61 & 8 & 120 & 22 & 9 & 98 & 14 & 292 & 24 & 10 & 59 & 42 & 31 & 1 & 5 \\
\hline$T A C T$ & 204 & 698 & 12412 & 333 & 1202 & 23 & 43 & 22 & 30 & 48 & 27 & 94 & 943 & 60 & 100 & 28 & 143 & 327 & 8 & 23 \\
\hline$R \mid A G A$ & 108 & 101 & 33 & 22 & 13 & 169 & 3 & 20 & 244 & 33 & 108 & 38 & 15 & 94 & 58 & 47 & 13 & 5 & 3 & 2 \\
\hline$S \mid A G C$ & 4025 & 1342 & 1741 & 344 & 112 & 64 & 47 & 8 & 57 & 460 & 68 & 151 & 412 & 3307 & 669 & 5 & 119 & 38 & 61 & 30 \\
\hline$R \mid A G G$ & 104 & 123 & 80 & 17 & 22 & 602 & 17 & 86 & 69 & 15 & 297 & 25 & 19 & 113 & 99 & 16 & 12 & 2 & 5 & 2 \\
\hline$S A G T$ & 1020 & 3570 & 328 & 72 & 297 & 49 & 9 & 10 & 49 & 136 & 69 & 392 & 70 & 724 & 1386 & 7 & 35 & 54 & 18 & 4 \\
\hline I $A T A$ & 16 & 16 & 93 & 2981 & 2224 & 23 & 79 & 339 & 53 & 4 & 35 & 5 & 36 & 6 & 3 & 1421 & 236 & 137 & 4 & 28 \\
\hline$I \mid A T C$ & 236 & 45 & 1429 & 95708 & 319908 & 5 & 1531 & 226 & 11 & 47 & 8 & 10 & 413 & 90 & 16 & 252 & 5416 & 909 & 32 & 522 \\
\hline$M \mid A T G$ & 75 & 58 & 370 & 854 & 862 & 120 & 178 & 2474 & 28 & 13 & 164 & 11 & 132 & 13 & 12 & 302 & 223 & 212 & 3 & 54 \\
\hline I $A T T$ & 63 & 167 & 286 & 16366 & 54789 & 8 & 241 & 149 & 12 & 9 & 9 & 32 & 96 & 32 & 42 & 140 & 869 & 2577 & 7 & 94 \\
\hline$Q C A A$ & 95 & 61 & 9 & 3 & 4 & 14988 & 56 & 182 & 909 & 71 & 339 & 43 & 12 & 18 & 15 & 13 & 2 & 1 & 46 & 28 \\
\hline $\mathrm{H}$ CAC & 798 & 320 & 52 & 24 & 4 & 1695 & 360 & 79 & 55 & 412 & 113 & 116 & 51 & 164 & 31 & 2 & 18 & 5 & 1241 & 82 \\
\hline$Q C A G$ & 130 & 131 & 24 & 8 & 7 & 148989 & 9119 & 1289 & 566 & 136 & 2483 & 110 & 32 & 43 & 25 & 13 & 9 & 15 & 102 & 12 \\
\hline HCAT & 226 & 653 & 17 & 9 & 24 & 1223 & 92 & 49 & 49 & 114 & 93 & 279 & 21 & 34 & 77 & 3 & 4 & 20 & 331 & 30 \\
\hline$P \mid C C A$ & 5 & 10 & 59 & 10 & 9 & 672 & 122 & 1091 & 65 & 4 & 33 & 6 & 119 & 7 & 7 & 31 & 8 & 13 & 3 & 17 \\
\hline$P \mid C C C$ & 72 & 38 & 740 & 52 & 29 & 222 & 685 & 289 & 11 & 30 & 15 & 11 & 788 & 49 & 18 & 9 & 88 & 18 & 59 & 108 \\
\hline$P \mid C C G$ & 4 & 4 & 48 & 7 & 3 & 721 & 90 & 1000 & 16 & 3 & 66 & 6 & 68 & 10 & 3 & 7 & 3 & 4 & 3 & 15 \\
\hline$P \mid C C T$ & 17 & 47 & 233 & 21 & 89 & 157 & 246 & 284 & 12 & 12 & 14 & 48 & 279 & 14 & 19 & 7 & 17 & 49 & 22 & 40 \\
\hline$R \mid C G A$ & 15 & 8 & 1 & 1 & 1 & 608 & 21 & 69 & 63 & 10 & 30 & 3 & 0 & 30 & 9 & 9 & 1 & 1 & 8 & 5 \\
\hline$R \mid C G C$ & 72 & 37 & 27 & 7 & 3 & 413 & 283 & 62 & 8 & 65 & 21 & 7 & 36 & 279 & 38 & 0 & 9 & 3 & 90 & 45 \\
\hline$R|C G G|$ & 17 & 13 & 3 & 2 & 4 & 3737 & 48 & 427 & 22 & 7 & 137 & 6 & 5 & 37 & 20 & 5 & 20 & 9 & 12 & 4 \\
\hline$R \mid C G T$ & 25 & 99 & 3 & 7 & 11 & 240 & 58 & 29 & 3 & 14 & 11 & 31 & 8 & 57 & 77 & 1 & 3 & 11 & 29 & 12 \\
\hline$L C T A$ & 10 & 3 & 10 & 72 & 49 & 130 & 2150 & 13178 & 16 & 0 & 6 & 0 & 15 & 2 & 2 & 227 & 33 & 23 & 3 & 97 \\
\hline$L \mid C T C$ & 42 & 15 & 126 & 1521 & 351 & 89 & 74979 & 10241 & 22 & 17 & 18 & 14 & 158 & 33 & 10 & 41 & 887 & 155 & 74 & 1850 \\
\hline$L C T G$ & 12 & 19 & 28 & 340 & 242 & 1221 & 119841 & 163737 & $7 \quad 14$ & 5 & 101 & 4 & 59 & 9 & 7 & 187 & 181 & 116 & 14 & 403 \\
\hline$L \mid C T T$ & 11 & 28 & 28 & 268 & 913 & 79 & 10926 & 6044 & 3 & 8 & 7 & 19 & 32 & 11 & 17 & 22 & 140 & 427 & 20 & 297 \\
\hline$E$ GAA & 177 & 163 & 35 & 6 & 4 & 579 & 13 & 22 & 80704 & 1875 & 25592 & 1845 & 91 & 118 & 96 & 154 & 24 & 39 & 27 & 10 \\
\hline$D G A C$ & 2014 & 716 & 147 & 56 & 30 & 170 & 17 & 6 & 2386 & 90508 & 3351 & 23213 & 659 & 1402 & 334 & 16 & 181 & 52 & 173 & 15 \\
\hline$E \mid G A G$ & 244 & 226 & 30 & 16 & 15 & 2591 & 21 & 86 & 27179 & 3295 & 152612 & 230 & 158 & 7 & 1 & 57 & 52 & 4 & 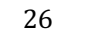 & 11 \\
\hline$D G A T$ & 605 & 1944 & 61 & 15 & 44 & 94 & 8 & 7 & 1769 & 20719 & 2606 & 70821 & 141 & 396 & 893 & 17 & 46 & 147 & 47 & 2 \\
\hline$A \mid G C A$ & 24 & 32 & 265 & 60 & 35 & 52 & 15 & 145 & 508 & 83 & 395 & 59 & 5329 & 69 & 74 & 696 & 127 & 128 & 2 & 13 \\
\hline$A \mid G C C$ & 170 & 75 & 3897 & 416 & 127 & 27 & 131 & 68 & 128 & 621 & 225 & 177 & 86827 & 7100 & 199 & 132 & 1969 & 392 & 25 & 111 \\
\hline$A \mid G C G$ & 11 & 13 & 248 & 49 & 28 & 91 & 11 & 149 & 145 & 44 & 485 & 34 & 4952 & 88 & 49 & 145 & 135 & 105 & 2 & 10 \\
\hline$A \mid G C T$ & 62 & 135 & 912 & 86 & 303 & 16 & 27 & 39 & 62 & 150 & 126 & 365 & 17318 & 280 & 437 & 106 & 432 & 1191 & 5 & 22 \\
\hline$G \mid G G A$ & 19 & 43 & 17 & 7 & 8 & 71 & 8 & 15 & 1247 & 125 & 434 & 102 & 70 & 4063 & 2444 & 126 & 25 & 19 & 2 & 3 \\
\hline$G \mid G G C$ & 452 & 183 & 195 & 91 & 27 & 47 & 31 & 14 & 110 & 1304 & 197 & 389 & 940 & 70299 & 10181 & 19 & 321 & 69 & 47 & 22 \\
\hline \begin{tabular}{|l|l|}
$G$ & $G G G$ \\
\end{tabular} & 39 & 66 & 30 & 11 & 10 & 239 & 6 & 97 & 334 & 115 & 1478 & 123 & 119 & 4951 & 3298 & 75 & 40 & 38 & 10 & 4 \\
\hline \begin{tabular}{|l|l|}
$G$ & $G G T$ \\
\end{tabular} & 91 & 343 & 44 & 27 & 59 & 24 & 8 & 1 & 91 & 330 & 125 & 862 & 200 & 10850 & 24402 & 16 & 61 & 199 & 17 & 4 \\
\hline$V$ GTA & 1 & 5 & 28 & 212 & 145 & 12 & 27 & 261 & 142 & 9 & 69 & 11 & 128 & 12 & 4 & 12620 & 1378 & 974 & 1 & 31 \\
\hline$V \mid G T C$ & 50 & 26 & 577 & 6081 & 1244 & 19 & 937 & 173 & 34 & 153 & 57 & 51 & 2028 & 234 & 50 & 1824 & 47252 & 6691 & 29 & 481 \\
\hline$V \mid G T G$ & 17 & 13 & 171 & 989 & 767 & 139 & 188 & 2165 & 102 & 23 & 498 & 26 & 562 & 57 & 49 & 8757 & 6074 & 4909 & 2 & 71 \\
\hline$V \mid G T T$ & 18 & 49 & 113 & 869 & 3036 & 7 & 127 & 96 & 20 & 43 & 30 & 138 & 437 & 86 & 195 & 1159 & 5514 & 24443 & 10 & 71 \\
\hline * TAA & 3 & 2 & 1 & 0 & 0 & 29 & 1 & 4 & 43 & 2 & 15 & 2 & 1 & 0 & 0 & 2 & 0 & 0 & 41 & 2 \\
\hline$Y T A C$ & 161 & 63 & 21 & 34 & 6 & 86 & 82 & 21 & 16 & 170 & 35 & 55 & 29 & 39 & 11 & 2 & 22 & 4 & 68411 & 941 \\
\hline * TAG & 2 & 5 & 0 & 1 & 1 & 207 & 3 & 35 & 12 & 1 & 89 & 1 & 0 & 1 & 0 & 0 & 0 & 0 & 28 & 1 \\
\hline$Y$ TAT & 55 & 209 & 13 & 7 & 26 & 76 & 26 & 17 & 16 & 73 & 26 & 169 & 5 & 3 & 32 & 0 & 11 & 10 & 16017 & 273 \\
\hline \begin{tabular}{|l|l|}
$S$ & $T C A$ \\
\end{tabular} & 16 & 10 & 134 & 4 & 5 & 74 & 23 & 203 & 44 & 3 & 37 & 5 & 152 & 6 & 3 & 54 & 15 & 8 & 15 & 75 \\
\hline \begin{tabular}{|l|l|}
$S$ & $T C C$
\end{tabular} & 108 & 26 & 1174 & 57 & 25 & 30 & 187 & 52 & 13 & 51 & 27 & 23 & 1690 & 51 & 7 & 8 & 108 & 26 & 261 & 725 \\
\hline \begin{tabular}{|l|l|}
$S$ & $T C G$
\end{tabular} & 7 & 12 & 103 & 12 & 8 & 71 & 10 & 151 & 10 & 4 & 39 & 5 & 138 & 7 & 4 & 9 & 6 & 2 & 9 & 51 \\
\hline \begin{tabular}{|l|l|}
$S$ & $T C T$
\end{tabular} & 20 & 83 & 325 & 23 & 67 & 28 & 49 & 66 & 6 & 14 & 20 & 51 & 470 & 37 & 54 & 11 & 32 & 57 & 103 & 258 \\
\hline * TGA & 1 & 1 & 0 & 0 & 0 & 27 & 1 & 12 & 14 & 3 & 10 & 1 & 1 & 12 & 4 & 4 & 1 & 0 & 3 & 3 \\
\hline \begin{tabular}{|l|l|}
$C$ & $T G C$ \\
\end{tabular} & 55 & 21 & 51 & 25 & 3 & 36 & 83 & 20 & 9 & 46 & 9 & 19 & 54 & 338 & 56 & 1 & 26 & 5 & 566 & 269 \\
\hline$W \mid T G G$ & 3 & 6 & 5 & 2 & 8 & 431 & 11 & 197 & 9 & 6 & 46 & 3 & 1 & 28 & 6 & 4 & 2 & 1 & 25 & 45 \\
\hline \begin{tabular}{|l|l|} 
& $T G T$ \\
\end{tabular} & 35 & 72 & 20 & 12 & 24 & 55 & 20 & 16 & 13 & 12 & 29 & 33 & 9 & 109 & 195 & 4 & 5 & 11 & 185 & 93 \\
\hline$L T T A$ & 0 & 2 & 10 & 43 & 39 & 20 & 291 & 1886 & 16 & 2 & 10 & 1 & 5 & 1 & 0 & 204 & 19 & 28 & 15 & 382 \\
\hline$F$ TTC & 18 & 10 & 72 & 505 & 105 & 13 & 1918 & 278 & 10 & 25 & 4 & 6 & 114 & 37 & 2 & 26 & 397 & 83 & 10429 & 92119 \\
\hline$L T T G$ & 7 & 1 & 23 & 75 & 80 & 130 & 918 & 15979 & 14 & 2 & 43 & 0 & 35 & 7 & 6 & 97 & 59 & 41 & 17 & 591 \\
\hline$F \mid T T T$ & 15 & 22 & 23 & 86 & 350 & 15 & 366 & 234 & 4 & 10 & 7 & 12 & 29 & 8 & 8 & 18 & 80 & 197 & 2871 & 16156 \\
\hline
\end{tabular}

Table 19. For the mammal gene multiple alignments with respect to the human reference genes $s_{1}=\mathbb{H}$, codon substitution matrix $\mathbf{A}(m(X, \mathbb{H}))$ of $X$ motifs $m(X, \mathbb{H})$ (Section 2.6). For each codon, the encoded amino acid is given. 
$\begin{array}{llllllllllllllllllllll}N & N & T & I & I & Q & L & L & E & D & E & D & A & G & G & V & V & V & Y & F\end{array}$

\begin{tabular}{lllllllllllllllllllll}
\hline$A A C$ & $A A T$ & $A C C$ & $A T C$ & $A T T$ & $C A G$ & $C T C$ & $C T G$ & $G A A$ & GAC & GAG & GAT & GCC & GGC & GGT & GTA & GTC & GTT & TAC & TTC \\
\hline
\end{tabular}

K $\mid \begin{array}{lllllllllllllllllllllll}1235 & 1785 & 475 & 304 & 592 & 769 & 107 & 187 & 2233 & 890 & 1083 & 1517 & 357 & 284 & 432 & 250 & 221 & 409 & 277 & 187\end{array}$ $\begin{array}{lllllllllllllllllllllllll}N & A A C & 985210627 & 507 & 238 & 394 & 477 & 82 & 136 & 1752 & 1456 & 764 & 2375 & 319 & 418 & 805 & 209 & 193 & 346 & 300 & 245\end{array}$ K $A A G \quad \begin{array}{llllllllllllllllllllll}1512 & 2008 & 612 & 364 & 595 & 959 & 117 & 227 & 2653 & 991 & 1280 & 1665 & 467 & 320 & 551 & 289 & 289 & 579 & 298 & 243\end{array}$ $\begin{array}{lllllllllllllllllllllll}N & A A T & 714310014 & 516 & 288 & 532 & 490 & 90 & 150 & 1931 & 1473 & 878 & 2699 & 319 & 453 & 821 & 251 & 186 & 338 & 304 & 214\end{array}$ $\begin{array}{llllllllllllllllllllll}T & A C A & 574 & 900 & 1923 & 320 & 462 & 215 & 70 & 105 & 771 & 344 & 420 & 638 & 278 & 141 & 251 & 267 & 283 & 451 & 130 & 178\end{array}$ $\begin{array}{llllllllllllllllllllll}T \text { ACC } & 622 & 880 & 3844 & 405 & 545 & 240 & 81 & 136 & 889 & 418 & 402 & 690 & 345 & 131 & 318 & 301 & 405 & 605 & 186 & 163\end{array}$ T ACG $28 \begin{array}{lllllllllllllllllllll} & 471 & 929 & 175 & 263 & 119 & 36 & 67 & 365 & 201 & 190 & 307 & 132 & 55 & 119 & 142 & 115 & 237 & 77 & 83\end{array}$

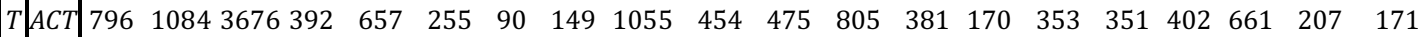
$\begin{array}{llllllllllllllllllllll}R & A G A & 491 & 677 & 171 & 140 & 225 & 276 & 50 & 94 & 622 & 233 & 334 & 471 & 119 & 114 & 211 & 123 & 116 & 172 & 155 & 115\end{array}$ $\begin{array}{lllllllllllllllllllllll}S & \text { AGC } & 581 & 823 & 274 & 118 & 185 & 160 & 42 & 62 & 699 & 391 & 338 & 649 & 187 & 179 & 278 & 120 & 95 & 180 & 103 & 104\end{array}$ $\begin{array}{llllllllllllllllllllll}R & A G G & 174 & 218 & 61 & 60 & 77 & 129 & 21 & 26 & 255 & 95 & 120 & 184 & 38 & 37 & 60 & 26 & 40 & 61 & 47 & 44\end{array}$ \begin{tabular}{l|llllllllllllllllllll}
$S$ & $A$ &
\end{tabular}

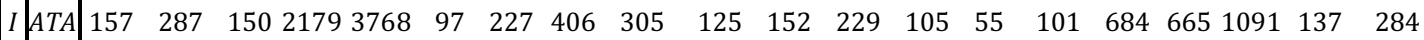
\begin{tabular}{l|l|llllllllllllllllll} 
& ATC & 287 & 331 & 292 & 745410862 & 131 & 350 & 601 & 398 & 192 & 221 & 327 & 214 & 75 & 127 & 107216592450 & 248 & 538
\end{tabular}

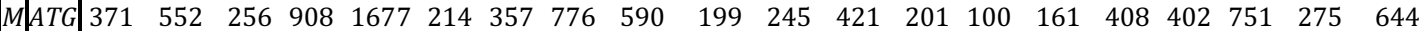

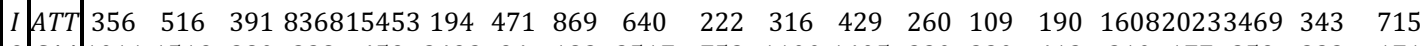

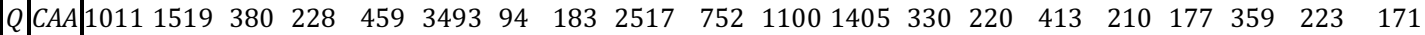

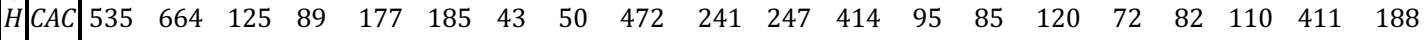

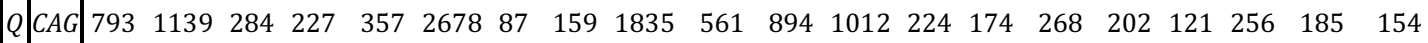
$\begin{array}{lllllllllllllllllllllllll}H & \text { CAT } & 640 & 928 & 167 & 116 & 227 & 270 & 60 & 73 & 622 & 323 & 309 & 583 & 92 & 127 & 181 & 118 & 96 & 143 & 454 & 225\end{array}$ $\begin{array}{lllllllllllllllllllllllllll}P & C C A & 416 & 637 & 216 & 156 & 290 & 185 & 52 & 73 & 767 & 388 & 330 & 672 & 261 & 115 & 271 & 127 & 131 & 239 & 103 & 109\end{array}$ $\begin{array}{llllllllllllllllllllll}P & C C C & 253 & 383 & 133 & 89 & 174 & 94 & 34 & 62 & 484 & 239 & 242 & 382 & 179 & 76 & 165 & 92 & 87 & 172 & 65 & 67\end{array}$ \begin{tabular}{ll|lllllllllllllllllllllll}
$P$ & $C C G$ & 127 & 252 & 60 & 38 & 91 & 58 & 14 & 34 & 238 & 134 & 109 & 230 & 75 & 40 & 82 & 53 & 52 & 73 & 47 & 39
\end{tabular} $\begin{array}{lllllllllllllllllllllll}P & C C T & 381 & 598 & 190 & 151 & 268 & 159 & 59 & 66 & 737 & 364 & 365 & 677 & 264 & 107 & 245 & 126 & 124 & 245 & 122 & 118\end{array}$ \begin{tabular}{l|llllllllllllllllllllllll}
$R$ & $C G A$ & 193 & 292 & 69 & 63 & 106 & 157 & 30 & 43 & 258 & 134 & 156 & 204 & 53 & 54 & 87 & 46 & 47 & 85 & 68 & 51
\end{tabular}

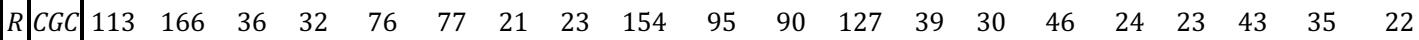
\begin{tabular}{l|lllllllllllllllllllll}
$R$ & $C G G$ & 99 & 136 & 46 & 39 & 53 & 64 & 3 & 19 & 120 & 66 & 72 & 110 & 23 & 22 & 50 & 22 & 19 & 38 & 42 & 18
\end{tabular} \begin{tabular}{l|llllllllllllllllllllll}
$R$ & $C G T$ & 211 & 299 & 75 & 55 & 106 & 127 & 22 & 39 & 319 & 160 & 133 & 250 & 51 & 54 & 102 & 47 & 42 & 82 & 68 & 43
\end{tabular} $\begin{array}{lllllllllllllllllllllll}L & C T A & 105 & 147 & 58 & 353 & 595 & 68 & 391 & 797 & 174 & 74 & 89 & 132 & 65 & 23 & 45 & 181 & 170 & 279 & 98 & 241\end{array}$ $\begin{array}{lllllllllllllllllllllll}L & C T C & 127 & 187 & 128 & 734 & 1021 & 89 & 573 & 1274 & 264 & 98 & 140 & 163 & 97 & 54 & 92 & 259 & 294 & 508 & 177 & 451\end{array}$

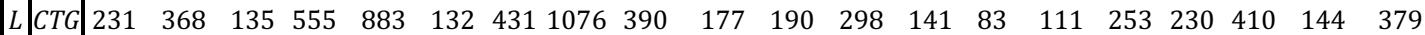
$\begin{array}{llllllllllllllllllllllllll}L & C T T & 230 & 338 & 166 & 959 & 1513 & 118 & 808 & 1749 & 368 & 166 & 182 & 270 & 150 & 76 & 105 & 397 & 418 & 709 & 248 & 572\end{array}$

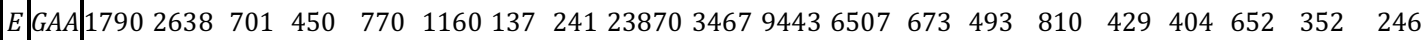

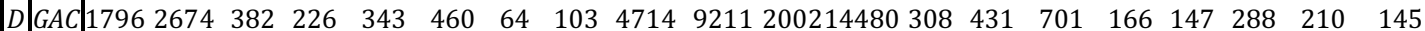
E $G A G\left[\begin{array}{lllllllllllllllllllllllllll}1246 & 1802 & 459 & 316 & 550 & 861 & 103 & 174 & 15702 & 2460 & 6558 & 4270 & 451 & 314 & 554 & 285 & 256 & 493 & 250 & 219\end{array}\right.$ D GAT $2463 \begin{array}{lllllllllllllllllllll}5633 & 306 & 438 & 616 & 119 & 151 & 6351 & 11838284221898 & 413 & 569 & 929 & 267 & 237 & 413 & 275 & 228\end{array}$ $\begin{array}{lllllllllllllllllllllllll}A & G C A & 481 & 651 & 364 & 284 & 445 & 207 & 69 & 114 & 879 & 348 & 426 & 707 & 2571 & 281 & 519 & 321 & 318 & 536 & 131 & 169\end{array}$ A GCC $5 \begin{array}{llllllllllllllllllllll}528 & 719 & 447 & 285 & 482 & 216 & 88 & 158 & 937 & 393 & 447 & 627 & 4330 & 303 & 796 & 319 & 408 & 610 & 166 & 166\end{array}$ $\begin{array}{llllllllllllllllllllllll}A & G C G & 180 & 283 & 116 & 114 & 193 & 103 & 38 & 61 & 333 & 135 & 167 & 267 & 946 & 114 & 233 & 142 & 112 & 199 & 53 & 79\end{array}$ \begin{tabular}{l|lllllllllllllllllllllllll}
$A$ & $G C T$ & 681 & 986 & 584 & 431 & 711 & 305 & 101 & 187 & 1339 & 590 & 621 & 926 & 5124 & 437 & 979 & 463 & 486 & 851 & 202 & 229
\end{tabular} G GGA $6 \begin{array}{llllllllllllllllllll}654 & 980 & 189 & 110 & 226 & 160 & 47 & 52 & 717 & 499 & 401 & 905 & 350 & 3102 & 8881 & 96 & 114 & 154 & 134 & 116\end{array}$ $\begin{array}{lllllllllllllllllllllll}G & G G C & 490 & 745 & 149 & 67 & 128 & 112 & 40 & 47 & 505 & 370 & 291 & 655 & 219 & 1922 & 4921 & 68 & 66 & 104 & 73 & 89\end{array}$

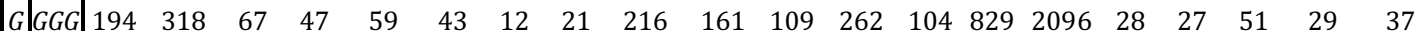
G $G$ GGT $8 \begin{array}{llllllllllllllllllll} & 807 & 1045 & 212 & 134 & 201 & 146 & 57 & 54 & 769 & 548 & 371 & 889 & 517 & 417321358 & 83 & 127 & 209 & 116 & 143\end{array}$

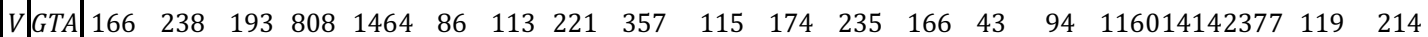

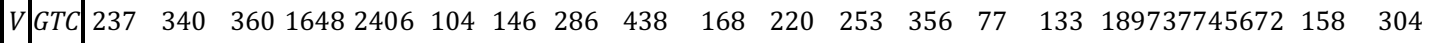

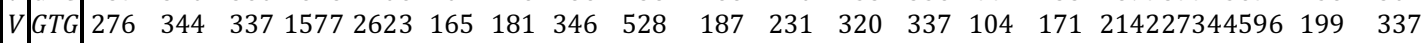

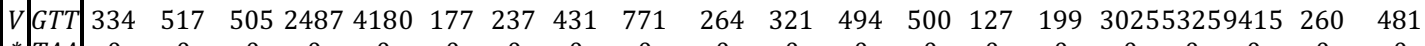

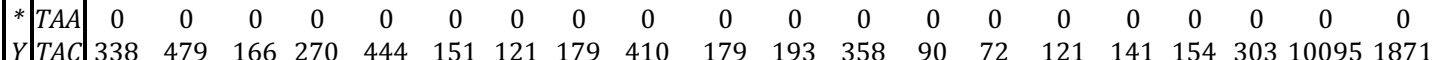

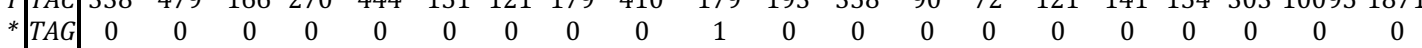

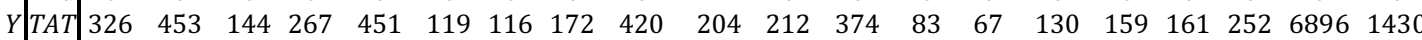

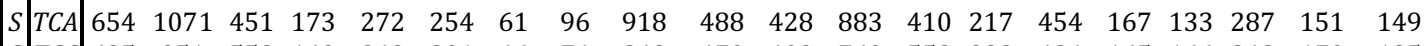
$\begin{array}{llllllllllllllllllllllllll}S & \text { TCC } & 635 & 951 & 558 & 149 & 249 & 201 & 46 & 74 & 849 & 470 & 402 & 760 & 558 & 228 & 424 & 165 & 166 & 248 & 170 & 125\end{array}$

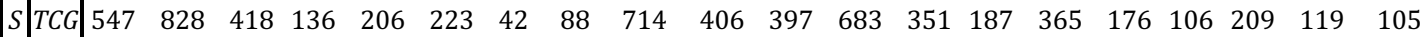
$S$
TTCT $\quad \begin{array}{lllllllllllllllllllllllll} & 1513 & 725 & 239 & 414 & 322 & 64 & 118 & 1430 & 740 & 636 & 1272 & 724 & 312 & 620 & 240 & 203 & 414 & 223 & 213\end{array}$

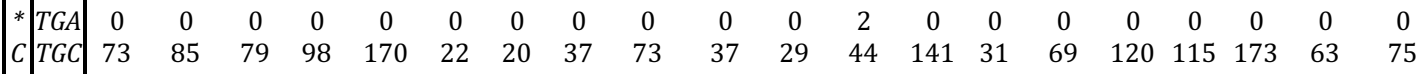

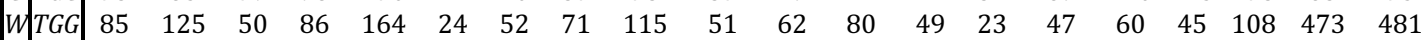
$\begin{array}{llllllllllllllllllllllll}\text { C } & \text { TGT } & 83 & 157 & 151 & 176 & 230 & 36 & 33 & 79 & 85 & 63 & 43 & 82 & 248 & 51 & 86 & 189 & 247 & 343 & 69 & 141\end{array}$ $\begin{array}{llllllllllllllllllllll}L & \text { TTA } & 194 & 318 & 154 & 855 & 1465 & 112 & 894 & 1970 & 332 & 167 & 181 & 252 & 146 & 52 & 110 & 411 & 374 & 694 & 197 & 575\end{array}$ F $\mid \begin{array}{lllllllllllllllllllllll} & 219 & 340 & 133 & 522 & 759 & 102 & 242 & 407 & 271 & 136 & 146 & 266 & 131 & 79 & 107 & 246 & 282 & 484 & 1598 & 11263\end{array}$ $\begin{array}{lllllllllllllllllllllll} & \end{array}$ \begin{tabular}{l|lllllllllllllllllllll}
$F$ & TTT & 299 & 453 & 162 & 579 & 1064 & 130 & 301 & 505 & 397 & 200 & 188 & 336 & 149 & 100 & 155 & 306 & 272 & 557 & 1725 & 10020 \\
\hline
\end{tabular}

Table 20. For the yeast gene multiple alignments with respect to the $S$. cerevisiae reference genes $s_{1}=\mathbb{C}$, codon substitution matrix $\mathbf{A}(m(X, \mathbb{C}))$ of $X$ motifs $m(X, \mathbb{C})$ (Section 2.6). For each codon, the encoded amino acid is given. 


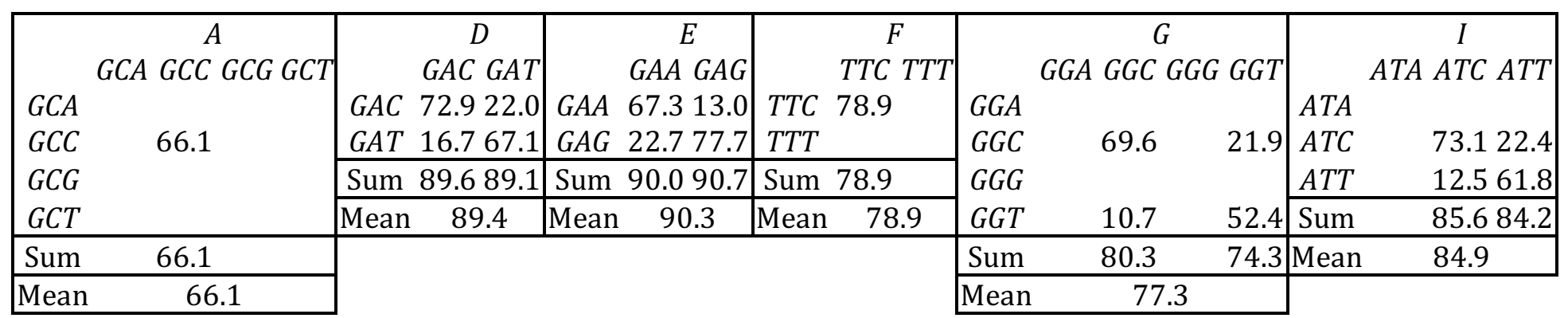

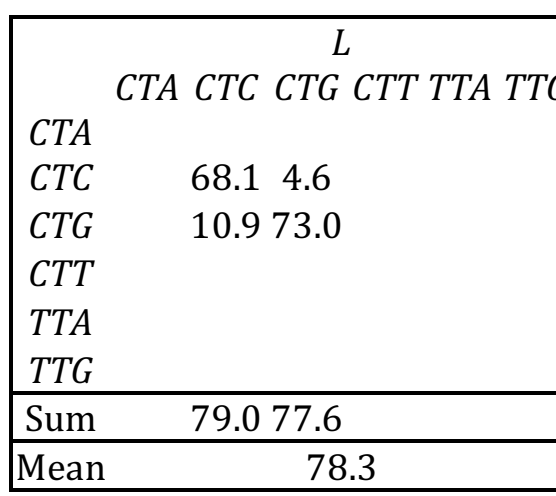

\begin{tabular}{|c|c|c|c|c|c|c|c|c|}
\hline$N$ & \multirow{2}{*}{\multicolumn{2}{|c|}{$\begin{array}{c}Q \\
C A A C A G\end{array}$}} & \multirow{2}{*}{\multicolumn{2}{|c|}{$\begin{array}{c}T \\
A C A \text { ACC } A C G \text { ACT }\end{array}$}} & \multirow{2}{*}{\multicolumn{3}{|c|}{$\begin{array}{c}V \\
\text { GTA GTC GTG GTT }\end{array}$}} & \multirow{2}{*}{$\begin{array}{c}Y \\
\text { TAC TAT }\end{array}$} \\
\hline$A A C A A T$ & & & & & & & & \\
\hline$A A C \quad 71.122 .2$ & $C A A$ & & $A C A$ & & GTA & $42.7 \quad 1.9$ & 2.1 & TAC 76.1 \\
\hline$A A T \quad 15.562 .3$ & $C A G$ & 80.7 & $A C C$ & 63.5 & $G T C$ & 6.264 .2 & 14.8 & TAT \\
\hline Sum 86.684 .5 & Sum & 80.7 & $A C G$ & & $G T G$ & & & Sum 76.1 \\
\hline Mean & Mean & 80.7 & $A C T$ & & GTT & 3.97 .5 & 53.9 & Mean \\
\hline & & & Sum & 63.5 & Sum & 52.873 .6 & 70.9 & \\
\hline & & & Mean & 63.5 & Mean & 65.7 & & \\
\hline
\end{tabular}

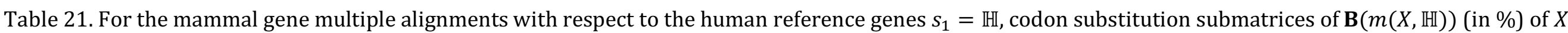
motifs $m(X, \mathbb{H})$ (Section 2.6) for the 12 amino acids $p \in \mathcal{X}=\{A, D, E, F, G, I, L, N, Q, T, V, Y\}$ coded by the circular code $X(1)$. 


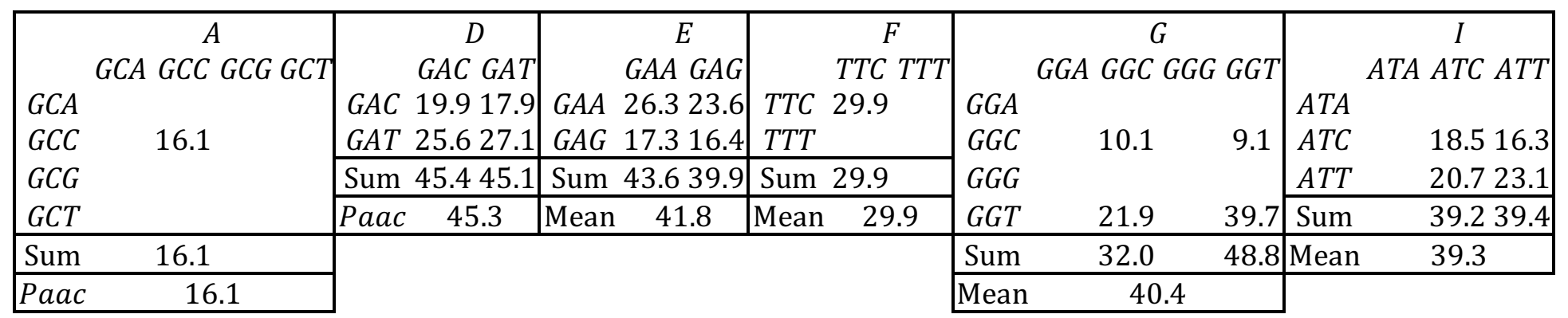

\begin{tabular}{|lrl|}
\hline \multicolumn{4}{c|}{ L } \\
CTA & CTC & CTG CTT TTA TTG \\
CTC & 5.6 & 6.2 \\
CTG & 4.2 & 5.2 \\
CTT & & \\
TTA & & \\
TTG & & \\
\hline Sum & 9.9 & 11.4 \\
\hline Mean & \multicolumn{2}{c|}{10.6} \\
\hline
\end{tabular}

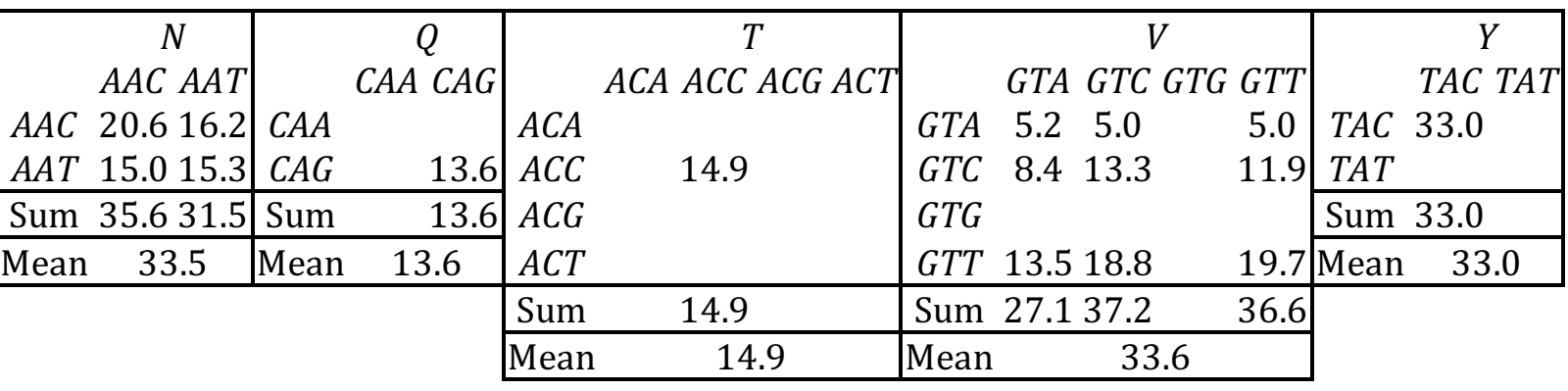

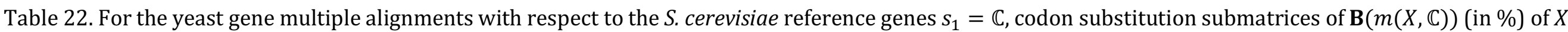
motifs $m(X, \mathbb{C})$ (Section 2.6) for the 12 amino acids $p \in \mathcal{X}=\{A, D, E, F, G, I, L, N, Q, T, V, Y\}$ coded by the circular code $X(1)$. 